\title{
On the General Theory of the Cauchy Type Functional Equations with Applications in Analysis.
}

\author{
B.Paneah (Technion, Israel)
}

\section{Introduction.}

In the last 30-40 years functional equations have grown to be a large, independent branch of mathematics with its own methods, circle of problems and, what is of great importance, abounding in applications. If on an early stage of development (XVIII - XIX centuries) functional equations played some auxiliary, may be even decorative role, describing in an abstract form various fundamental functions from Analysis, then nowadays functional equations turn out to be a powerful tool when solving analytical problems in quite different fields of mathematics. One of the first and most important functional equations was considered by Cauchy. He formulated the following problem (in a local form): find all continuous function $F$ on the interval $\{z \mid-1 \leq z \leq 1\}$ such that the relation

$$
F(x+y)-F(x)-F(y)=0
$$

valid for all points $(x, y)$ in the square

$$
K=\{(x, y)|| x \pm y \mid \leq 1\}
$$

As is well known, the only linear functions $F(z)=\lambda z, \lambda$ is a constant, solve this problem. It looks surprisingly, but as was clarified recently, a great deal of various problems in such diverse fields as integral and functional equations, measure theory, integral geometry, boundary problems for hyperbolic partial differential equations turn out to be reducible (sometimes in an equivalent manner) to the functional equation

$$
F\left(\left(\delta_{1}+\delta_{2}\right)(t)\right)-F\left(\delta_{1}(t)\right)-F\left(\delta_{2}(t)\right)=h(t), \quad t \in I=(-1,1),
$$

essentially coinciding with Cauchy equation (0.1). Here $\delta_{1}$ and $\delta_{2}$ are given continuous maps of the interval $I$ into itself, and $h$ and $F$ are some given and unknown functions with corresponding domains. Precisely this equation is the main object of the present paper. It is worth adding that equation (0.2) can be regarded as an analog of the famous cohomological equation

$$
F(t)-F(\delta(t))=h(t)
$$

appearing in connection with many problem of the theory of dynamical systems and ergodic theory. Thus, equation (0.2) is of interest not only by itself as an object of analysis. It is also a necessary link in solving various problems in diverse fields that, at first sight, by no means have any connection with functional equations. It turns out that the solvability properties of equation (0.2) and the methods of their deriving are determined by some mutual properties of the functions $\delta_{j}(t), j=1,2$. These mutual properties are formalized by means of a "configuration" formed by the functions $\delta_{j}$. As will be seen below, when studying equation (0.2) in the case of a $Z$ - 
configuration, we arrive at the explicit (!) solution of the equation, remaining within frameworks of pure analysis. But the same equation in the case of a $\mathcal{P}$ - configuration requires using some delicate results from functional analysis and also applying new dynamical methods. The application of these methods becomes possible if we associate equation (0.2) with the semigroup $\Phi_{\delta}$ of maps from an interval $I$ into itself generated by $\delta_{1}$ and $\delta_{2}$. Not only all the main results related to solvability properties are formulated in terms of orbits of this semigroup, but also their proofs are based on the existence of some specific attractors of the corresponding dynamical system. All these results and methods are the subject of this paper. A special attention is given to the applications of the results in functional equations in Integral geometry and in PDE. The existence of such deep and unexpected connection between these parts of analysis is the main novelty related to the general theory of functional equations. It would be interesting, by my opinion, to clarify, whether some other functional equations different from equation (0.1), are also closely connected with some analytical problems of quite different form.

\section{The main notations and definitions.}

In this paper we deal mostly with two intervals

$$
I=\{t \mid-1 \leq t \leq 1\} \text { and } I^{\prime}=\{t \mid 0 \leq t \leq 1\} .
$$

Definition 1. Given some real-valued functions $H$ and $\alpha_{1}, \alpha_{2}$ on the interval $\mathcal{I}=I$ or $I^{\prime}$, the equation

$$
F\left(\left(\alpha_{1}+\alpha_{2}\right)(t)\right)-F\left(\alpha_{1}(t)\right)-F\left(\alpha_{2}(t)\right)=H(t), \quad t \in \mathcal{I}
$$

with $F$ being an unknown real-valued function with the domain

$$
\mathcal{D}_{F}=\mathcal{R}\left(\alpha_{1}+\alpha_{2}\right) \cup \mathcal{R}\left(\alpha_{1}\right) \cup \mathcal{R}\left(\alpha_{2}\right)
$$

is said to be Cauchy type functional equation on $\mathcal{I}$.

Introducing a linear operator $\mathcal{B}_{\alpha}: C(\mathcal{I}) \rightarrow C\left(\mathcal{D}_{F}\right)$ of the form

$$
\mathcal{B}_{\alpha}: F(t) \mapsto F\left(\alpha_{1}(t)+\alpha_{2}(t)\right)-F\left(\alpha_{1}(t)\right)-F\left(\alpha_{2}(t)\right)
$$

makes it possible to rewrite (1) in a short form as

$$
\mathcal{B}_{\alpha} F(t)=H(t), \quad t \in \mathcal{I} .
$$

As will be seen, both solvability properties of this equation and the methods of their receiving depend essentially on some mutual properties (configurations) of the functions $\alpha_{1}$ and $\alpha_{2}$. In this paper we restrict ourselves to two different configurations formed by $\alpha_{1}$ and $\alpha_{2}$, and we fix this difference in the following definitions.

Definition 2. We say that the maps $\alpha_{1}$ and $\alpha_{2}$ from $I$ into itself form a $\mathcal{P}$ configuration if

$1^{\circ} \quad$ both functions do not decrease on $I$;

$2^{\circ} \quad$ the ranges of the maps $\alpha_{1}$ and $\alpha_{2}$ are the closed intervals $[0,1]$

and $[-1,0]$, respectively.

It follows that

$$
\alpha_{1}(-1)=\alpha_{2}(1)=0, \quad \alpha_{1}(1)=1, \quad \alpha_{2}(-1)=-1 .
$$

Definition 3. We say that real functions $\alpha_{1}(t)$ and $\alpha_{2}(t)$ on the interval $I^{\prime}$ form a $Z$ - configuration if 
Figures 1 and 2 represent typical examples of $Z$ - and $\mathcal{P}$ - configurations, respectively. Dotted lines in both figures are the graphs of the functions $z=\alpha_{1}(t)+\alpha_{2}(t)$ in $(t, z)$ - plane.

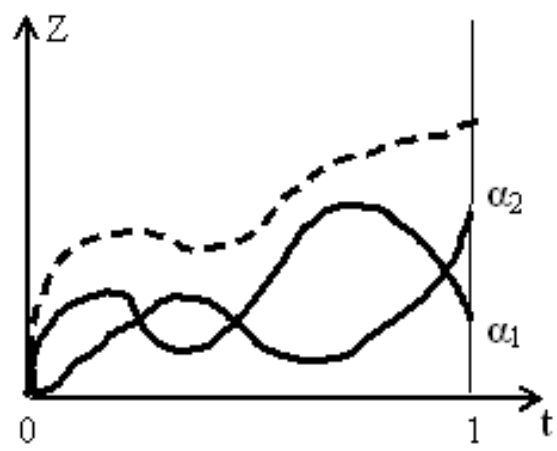

Fig. 1

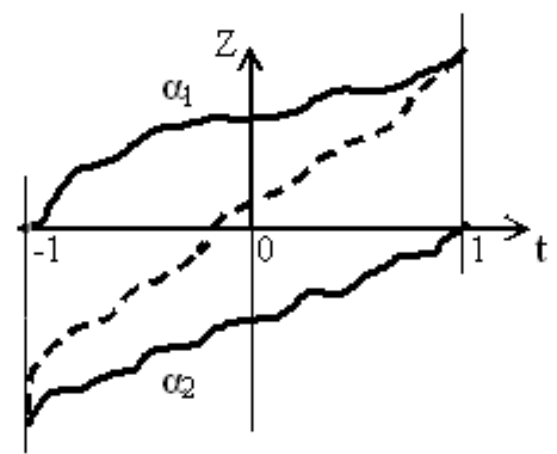

Fig.2

We note that the above conditions determining the two configurations are not artificial in the sense that all functional equations of the type (1) that have up to now arisen in various problems in geometry, in the theory of integral and functional equations, and also in the theory of boundary problems for hyperbolic partial differential equations satisfy these conditions (see [P1] - [P3]). Some confirmations of this fact the reader will find in Sec. 4.

It turns out that an essential information related to the solvability of equation (1), especially, in the case of a $\mathcal{P}$ - configuration, may be derived by means of new dynamical methods, introduced in the author's papers [P5]. The application of these methods becomes possible if we associate this equation with a noncommutative semigroup of maps from the interval $\mathcal{I}$ into itself, generated by $\alpha_{1}$ and $\alpha_{2}$. Pass to the exact formulations.

Definition 4. Given maps $\alpha_{1}, \alpha_{2}$, from the interval $\mathcal{I}$ into itself we denote by $\Phi_{\alpha}$ the noncommutative semigroup of maps from $\mathcal{I}$ into itself generated by these maps. The elements of this semigroup are all maps from $\mathcal{I}$ into itself of the form

$$
\alpha_{J_{\langle 0\rangle}}=i d, \alpha_{J_{\langle n\rangle}}=\alpha_{j_{n}} \circ \cdots \circ \alpha_{j_{1}}, \quad n=1,2, \ldots,
$$

where $J_{\langle n\rangle}=\left(j_{1}, \ldots, j_{n}\right)$ is an arbitrary $n$ - tuple with all $j_{k}=1,2$, and $\circ$ denotes the composition of maps. This semigroup determines naturally a dynamical system.

When dealing with a $Z$ - configuration and the corresponding functions $\alpha_{j}$ are defined on the interval $I^{\prime}$, it is convenient to work with the spaces

$$
C_{\langle k+r\rangle}\left(I^{\prime}\right)=\left\{u(t) \mid u(t)=P_{k}(t)+t^{k+r} \phi(t)\right\},
$$

where $k \geq 1$ is an integer, and $|r|<1$. Here $P_{k}(t)$ is an arbitrary polynomial of degree $\leq k$, and $\varphi(t)$ is an arbitrary continuous functions on $I^{\prime}$ if $r>0$ and, in addition, $\varphi(0)=0$, if $r=0$. With the norm

$$
\|u\|_{\langle k+r\rangle}=\sup _{I^{\prime}}\left|P_{k}(t)\right|+\sup _{I^{\prime}}|\phi(t)|
$$

the space $C_{\langle k+r\rangle}\left(I^{\prime}\right)$ becomes a Banach space. Roughly speaking, elements of this space are continuous functions on $I^{\prime}$ whose derivative $u^{(k)}$ at the point $t=0$ satisfy the Hölder condition of order $r$. It is obvious that $C_{\langle k-r\rangle}\left(I^{\prime}\right) \supset C_{\langle k\rangle}\left(I^{\prime}\right) \supset C_{\langle k+r\rangle}\left(I^{\prime}\right)$. 


\section{Statement of the problems and main results.}

\subsection{The case of a $Z$-configuration.}

In this subsection we deal with the functional equation (1), where $t \in I^{\prime}$, and the functions $\alpha_{1}, \alpha_{2}$ form a $Z$-configuration. It is assumed, unless otherwise stated, that $\alpha_{j}(t) \in C_{\langle 1\rangle}\left(I^{\prime}\right), j=1,2$. As to an unknown function $F$, it has to be defined on the interval $\widehat{I}=\{t \mid 0 \leq t \leq \tau\}$ with $\tau=\max _{t \in I^{\prime}} \sum_{j} \alpha_{j}(t)$. It is obvious that the homogeneous equation $\mathcal{B}_{\alpha} F=0$ has nontrivial solutions $F(t)=\lambda t, \lambda \in \mathbb{R}$. On the other hand, differentiating equation (1) at the point $t=0$ leads to the relation (independently of $F !$ )

$$
H^{\prime}(0)=0,
$$

which is thus a necessary condition for solvability of this equation. These observations enable us to formulate the following problem for equation (1) which is (as will be seen) well posed.

Given real number $\lambda$ and a function $H \in C_{\langle 1\rangle}\left(I^{\prime}\right)$, satisfying conditions (3) find a function $F \in C_{\langle 1\rangle}(\widehat{I})$ such that

$$
\mathcal{B}_{\alpha} F=H \text { on } I^{\prime}, \quad F^{\prime}(0)=\lambda .
$$

The exposition of the results related to this problem we begin with the theorem which describes an unexpected connection of the dynamical system generated by the semigroup $\Phi_{\alpha}$ with some classical structures of analysis, on the one hand, and on the other hand, with the Cauchy equation $F(x+y)-F(x)-F(y)=0$, which is undoubtedly one of fundamental equations in analysis.

Theorem $11^{\circ} \quad$ Let functions $\alpha_{1}(t)$ and $\alpha_{2}(t)$ satisfy the hypotheses

$$
\alpha_{1}(t)+\alpha_{2}(t)=t, \quad t \in I^{\prime}
$$

and

$$
\alpha_{1}^{\prime}(0) \alpha_{2}^{\prime}(0) \neq 0 \text {. }
$$

If both functions belong to the space $C_{\langle 1+r\rangle}\left(I^{\prime}\right), 0<r<1$, then, for an arbitrary function $H \in C_{\langle 1+r\rangle}\left(I^{\prime}\right)$ satisfying the boundary conditions

$$
H(0)=H^{\prime}(0)=0,
$$

the series

$$
\sum_{J} H\left(\alpha_{J}(t)\right), \quad t \in I
$$

with the summation over all multi-indices $J=J_{\langle n\rangle}, n=0,1, \ldots$, converges on $I^{\prime}$ absolutely and uniformly.

$2^{\circ} \quad$ The sum $F(t)$ of this series is a solution of equation $\mathcal{B}_{\alpha} F=H$ and belongs to the space $C_{\langle 1+r\rangle}\left(I^{\prime}\right)$.

Thus, under condition (5) the relation

$$
F(t)=\sum_{J} H\left(\alpha_{J}(t)\right)
$$

determines a solution to equation (1) in an explicit form. In the theory of dynamical systems it is usual to call such series orbital series corresponding to the dynamical system $\Phi_{\alpha}$.

The complete solution of problem (4) is given in the following two theorems. 
Theorem 2 If the functions $\alpha_{1}$ and $\alpha_{2}$ satisfy condition (6) and $H=0$ on $I^{\prime}$, then, for a given real number $\lambda$, the linear function $F(t)=\lambda t$ is a unique solution of the problem (4) lying in the space $C_{\langle 1\rangle}\left(I^{\prime}\right)$.

It is of great importance to note that this uniqueness result is established, in contrast to the previous theorem, in the wider space $C_{\langle 1\rangle}\left(I^{\prime}\right)$ and without any additional hypotheses, related to the function $\alpha=\alpha_{1}+\alpha_{2}$.

Theorem 3 Assume that, in addition to condition (6) the function $\alpha$ is, in contrast with (5), invertible and the inverse function $\alpha^{-1}$ is continuous. Then, for an arbitrary function $H \in C_{\langle 1+r\rangle}\left(I^{\prime}\right)$, satisfying condition (3), and for any real number $\lambda$, there is a unique solution $F \in C_{\langle 1+r\rangle}\left(I^{\prime}\right)$ of problem (4).

Thus, by using the operator terminology, the linear operator $\mathcal{B}_{\alpha}$ is a Fredholm operator: $C_{\langle 1+r\rangle}\left(I^{\prime}\right) \rightarrow C_{\langle 1+r\rangle}(\widehat{I})$ of index zero.

The following theorem relates to the regularity of the solution $F$ to problem (4).

Assume that the functions $\alpha_{j}(t), j=1,2$, lie in the space $C^{k}\left(I^{\prime}\right)$ of $k$ times continuously differentiable functions, $k=1,2, \ldots$

Theorem 4 If the functions $\alpha_{1}(t)$ and $\alpha_{2}$ satisfy hypotheses (5) and (6), and $H \in$ $C^{k}\left(I^{\prime}\right), k=1,2, \ldots$, then all solutions of the equation $\mathcal{B}_{\alpha} F=H$ belong to the space $C^{k}\left(I^{\prime}\right)$. If the function $H(t)$ satisfies hypothesis (7), then the series $\sum_{J} H\left(\alpha_{J}(t)\right)$ converges in the topology of $C^{k}\left(I^{\prime}\right)$.

This result plays a crucial role when applying the technique of functional equations in the theory of boundary problems for partial differential equations (where such an result is usually said to be increasing in smoothness ) (see Sec. 4).

\subsection{The case of a $\mathcal{P}$-configuration.}

In this subsection we deal with the Cauchy type functional equation $\mathcal{B}_{\alpha} F=$ $H(t)$, but now $t \in I$ and the functions $\alpha_{1}(t)$ and $\alpha_{2}(t)$ form a $\mathcal{P}$-configuration. As it follows from Definition 2, for an arbitrary function $F \in C(I)$, the relation

$$
\left(\mathcal{B}_{\alpha} F\right)(-1)=\mathcal{B}_{\alpha} F(1)=-F(0)
$$

holds. It follows that the periodicity condition

$$
H(-1)=H(1)
$$

is necessary for solvability of the equation in question and, hence, the cokernel coker $\mathcal{B}_{\alpha}$ of the operator $\mathcal{B}_{\alpha}$ is not empty. As the kernel ker $\mathcal{B}_{\alpha}$ is also nonempty (as we already know), the best possible result related to the solvability of equation (1) is the fredholmness of $\mathcal{B}_{\alpha}$ and, in addition, the relations dimker $\mathcal{B}_{\alpha}=\operatorname{dim} \operatorname{coker} \mathcal{B}_{\alpha}=1$. This result will be achieved, if we prove the unique solvability of the following boundary problem:

given a number $\lambda \in \mathbb{R}$ and a function $H$ satisfying hypothesis (9), to find a function $F$ on $I$ such that

$$
\mathcal{B}_{\alpha} F=H \quad \text { on } I \quad \text { and } \quad F(1)=\lambda .
$$

The conditions for such solvability are related to smoothness of the functions $F$ and $H$, and to some nontrivial features of the dynamical system generated by the semigroup $\Phi_{\alpha}$. The following notions were introduced in [P1]. 
1) An ordered set $\mathcal{O}=\left(t_{1}, \ldots, t_{k+1}\right)$ of points in $I$ is said to be orbit (or orbit of the point $t_{1}$ ) generated by $\Phi_{\alpha}$ if

$$
t_{k+1}=\alpha_{j_{k}}\left(t_{k}\right), \quad 1 \leq k \leq n,
$$

for an arbitrary natural $n \geq 1$.

2) The subsets in $I$

$$
\mathcal{T}_{1}=\left\{t \mid \alpha_{2}^{\prime}(t)=0\right\}, \quad \mathcal{T}_{2}=\left\{t \mid \alpha_{1}^{\prime}(t)=0\right\}, \quad \mathcal{T}=\mathcal{T}_{1} \cup \mathcal{T}_{2}
$$

are said to be guiding sets.

3) An orbit $\mathcal{O}=\left(t_{1}, \ldots, t_{n+1}\right), n=1,2, \ldots$ is said to be $\mathcal{T}$ - proper if in (11) $\alpha_{j_{k}}=\alpha_{1}$, when $t_{k} \in \mathcal{T}_{1}$, and $\alpha_{j_{k}}=\alpha_{2}$, when $t_{k} \in \mathcal{T}_{2}$.

4) If all points of an orbit $\mathcal{O}$ belong to the guiding set $\mathcal{T}$, then $\mathcal{O}$ is said to be $\mathcal{T}$ - guided orbit.

5) If the end points of an orbit $\mathcal{O}=\left(t_{1}, \ldots, t_{n+1}\right)$ coincide, i.e. $t_{1}=t_{n+1}$, then this orbit is said to be periodic orbit or cycle.

Definition. We denote by $\mathfrak{N}_{\alpha}^{\mathcal{T}}$ the set of all $\mathcal{T}$ - proper $\mathcal{T}$ - guided periodic orbits in $I$.

Now everything is ready to formulate the main results treated the solvability properties of equation (1) in the case of a $\mathcal{P}$-configuration.

Denote by $\mathcal{T}_{j}^{\prime}$ the set of all limit points of the set $\mathcal{T}_{j}, j=1,2$, and let

$$
\tau_{1}=\min \left\{t \mid t \in \mathcal{T}_{1}^{\prime}\right\}, \quad \tau_{2}=\max \left\{t \mid t \in \mathcal{T}_{2}^{\prime}\right\}
$$

Theorem 5 Assume that the functions $\alpha_{1}(t)$ and $\alpha_{2}(t)$ belong to $C^{1}(I)$ and satisfy inequalities

$$
\alpha_{1}^{\prime}(t)+\alpha_{2}^{\prime}(t)>0 \quad \text { on } \stackrel{\circ}{I} \text { and } \quad \alpha_{1}^{\prime}(-1) \alpha_{2}^{\prime}(1)>0
$$

Let also

$$
\tau_{2}<\tau_{1},
$$

if both sets $\mathcal{T}_{1}$ and $\mathcal{T}_{2}$ are infinite, and let $\mathcal{T}_{j}$ be an arbitrary set in $I$, if the set $\mathcal{T}_{j^{\prime}}, j^{\prime} \neq j$, is finite. Then, all solutions of the homogeneous equation

$$
F\left(\alpha_{1}(t)+\alpha_{2}(t)\right)-F\left(\alpha_{1}(t)\right)-F\left(\alpha_{2}(t)\right)=0
$$

are linear functions $F(z)=\lambda z$, if

$$
\mathfrak{N}_{\alpha}^{\mathcal{T}}=\emptyset
$$

The assertion of the theorem remains true if the condition $F(1)=\lambda$ in (10) is replaced by the condition $F^{\prime}(0)=\lambda$. This leads to some insignificant changes in the proof.

Theorem 6 Assume that in addition to hypotheses (12) and (13) both functions $\alpha_{1}(t)$ and $\alpha_{2}(t)$ belong to $C^{2}(I)$. Then, for all real $\lambda$ and for an arbitrary function $H \in C^{2}(I)$, satisfying condition (9), there is a unique solution $F \in C^{2}(I)$ of problem (10) if and only if

$$
\mathfrak{N}_{\alpha}^{\mathcal{T}}=\emptyset .
$$

The inverse operator $h \mapsto F$ from the space $C^{2}(I)$ into itself is continuous. 


\section{Proofs of Theorems 1-7.}

\subsection{Proof of Theorem 1.}

$1^{\circ}$ Set $F(t)=\sum_{J} H\left(\alpha_{j}(t)\right)$. The function $H(t)$, as it follows from condition (7) and from the definition of the space $C_{\langle 1+r\rangle}\left(I^{\prime}\right)$, can be represented as $H(t)=t^{1+r} \varphi(t)$ with $\varphi(t)$ being a continuous function on $I^{\prime}$. For this reason,

$$
F(t)=\sum_{J} \alpha_{J}^{1+r}(t) \varphi\left(\alpha_{J}(t)\right)=\sum_{n=0}^{\infty} \sum_{J_{\langle n\rangle}} \alpha_{J_{\langle n\rangle}}^{1+r}(t) \varphi\left(\alpha_{J_{\langle n\rangle}}(t)\right) .
$$

Introduce the notation

$$
A_{n}(t)=\sum_{J_{\langle n\rangle}} \alpha_{J_{\langle n\rangle}}^{1+r}(t), \quad n=1,2, \ldots
$$

Taking into account the relation $\alpha_{J_{\langle 0\rangle}}(t)=t$ we can rewrite the function $A_{n}(t)$ in the form

$$
A_{n}(t)=t^{1+r} \sum_{j_{1}, \ldots, j_{n}=1}^{2}\left(\frac{\alpha_{j_{n}} \circ \ldots \circ \alpha_{j_{1}}(t)}{\alpha_{j_{n-1}} \circ \ldots \circ \alpha_{j_{1}}(t)}\right)^{1+r} \circ \ldots \circ\left(\frac{\alpha_{j_{1}}(t)}{t}\right)^{1+r} .
$$

Note that, by relations $3^{\circ},(5)$ and (6) there is a constant $q<1$ such that the inequality

$$
\left(\alpha_{1}(t) / t\right)^{1+r}+\left(\alpha_{2}(t) / t\right)^{1+r} \leq q
$$

holds for all $t \in I$. Therefore, for an arbitrary value $k, 1 \leq k \leq n$, we have

$$
\sum_{j_{k}=1}^{2}\left(\alpha_{j_{k}} \circ \ldots \circ \alpha_{j_{1}}(t) / \alpha_{j_{k-1}} \circ \ldots \circ \alpha_{j_{1}}(t)\right)^{1+r} \leq q
$$

where $\alpha_{j_{0}}=i d$. This leads immediately to the relation $A_{n}(t) \leq t^{1+r} q^{n}$ which implies the uniform convergence of the series $\sum_{J}\left(\alpha_{J}(t) / t\right)^{1+r}$. This results in the representation of the function $F(t)$ in the form

$$
F(t)=t^{1+r} f(t), \quad t \in I^{\prime},
$$

with $f(t)$ a continuous function, and completes the proof of assertion $1^{\circ}$.

$2^{\circ} \quad$ By virtue of relation (5) it can be directly shown that the function $F(t)$ satisfies the Cauchy type functional equation (1). This completes the proof of Theorem 1.

\subsection{Proof of Theorem 2.}

Dividing both parts of the homogeneous equation (1) by $\left(\alpha_{1}+\alpha_{2}\right)(t)$, we arrive at the equivalent functional equation

$$
\Phi\left(\alpha_{1}(t)+\alpha_{2}(t)\right)-\rho_{1}(t) \Phi\left(\alpha_{1}(t)\right)-\rho_{2}(t) \Phi\left(\alpha_{2}(t)\right)=0, \quad t \in I^{\prime},
$$

where

$$
\begin{array}{ll}
\Phi(z)=F(z) / z, & \rho_{j}(z)=\alpha_{j}(z) /\left(\alpha_{1}+\alpha_{2}\right)(z), \quad z \in I^{\prime} \backslash\{0\} \\
\Phi(0)=0, & \rho_{j}(0)=\alpha_{j}^{\prime} /\left(\alpha_{1}+\alpha_{2}\right)^{\prime}(0), \quad j=1,2,
\end{array}
$$

and all the functions $\alpha_{j}, \rho_{j}, \Phi$ are continuous on the interval $I^{\prime}$. Show that any continuous solution $\Phi$ of equation (16) is a constant. Indeed, let $\mathcal{M}=\max _{I^{\prime}} \Phi$ and 
let $t_{0}=\inf \{t \mid \Phi(t)=\mathcal{M}\}$. Assume that $t_{0}>0$. Then, by continuity, $\Phi\left(t_{0}\right)=\mathcal{M}$, and, therefore, the relation

$$
\Phi\left(\alpha_{1}\left(t_{1}\right)\right)=\Phi\left(\alpha_{2}\left(t_{1}\right)\right)=\mathcal{M},
$$

is valid for an arbitrary point $t_{1}$ belonging to the nonempty subset $\left\{t \mid \alpha_{1}(t)+\right.$ $\left.\alpha_{2}(t)=t_{0}\right\}$, as $\rho_{1}(t)+\rho_{2}(t)=1$ for all values $t \in I^{\prime}$. However, this contradicts to the definition of the point $t_{0}$, as $\alpha_{1}\left(t_{1}\right)<t_{0}$ (by Definition 3). Thus, we have proved that $t_{0}=0$, and hence, $\Phi(0)=\mathcal{M}$. Repeating literally these arguments in connection with the point of minimum of the function $\Phi$, we arrive at the relation $\Phi(0)=\min _{I^{\prime}} \Phi$, and this proves the theorem.

\subsection{Proof of Theorem 3.}

The result follows immediately from the previous two theorems. To see this, it suffices to change variable $\alpha(t) \rightarrow t$. Then we arrive at the functional equation

$$
G(t)-G\left(\rho_{1}(t)\right)-G\left(\rho_{2}(t)\right)=\widehat{H}(t), \quad t \in \widehat{I},
$$

where

$$
\rho_{1}(t)=\alpha_{1} \circ \alpha^{-1}(t), \quad \rho_{2}(t)=\alpha_{2} \circ \alpha^{-1}(t), \quad \widehat{H}(t)=H \circ \alpha^{-1}(t)
$$

and $\widehat{I}=(0, r), r=\max _{I^{\prime}}\left(\alpha_{1}(t)+\alpha_{2}(t)\right)$. It is clear that the above change of variable preserves the $C_{\langle 1+r\rangle^{-}}$smoothness of all involving functions, and, in addition, the functions $\rho_{j}(t), j=1,2$ and $H(t)$ satisfy conditions (5) and (3), respectively, on $\widehat{I}$. If we represent the write-hand side $\widehat{H}(t)$ in the form $\widehat{H}(t)=\widehat{H}(0)+\mathcal{H}(t)$, then the function $\mathcal{G}(t)=G(t)+\widehat{H}(0)$ is a solution of the equation

$$
\mathcal{B}_{\rho} \mathcal{G}:=\mathcal{G}(t)-\mathcal{G}\left(\rho_{1}(t)\right)-\mathcal{G}\left(\rho_{2}(t)\right)=\mathcal{H}(t)
$$

By Theorem 1 the function

$$
\mathcal{G}(t)=\sum_{J} \mathcal{H}\left(\rho_{J}(t)\right)
$$

solves this equation, and using Theorem 2 we find that the function

$$
G(t)=-\widehat{H}(0)+\sum_{J} \mathcal{H}\left(\rho_{J}(t)\right)+\lambda t
$$

is a unique (in the space $\left.C_{\langle 1+r\rangle}(\widehat{I})\right)$ solution of the problem

$$
\mathcal{B}_{\rho} G=\widehat{H} \quad \text { on } \widehat{I}, \quad G^{\prime}(0)=\lambda \alpha^{\prime}(0) .
$$

It remains to substitute $\alpha(t)$ for $t$ in (17), and to obtain the solution to problem (4) in an explicit form.

\subsection{Proof of Theorem 4.}

For the sake of brevity we restrict ourselves to the case $k=2$. The general case can be considered by an interested reader without any difficulty, as the proof below includes all the essential features of the general case. Note, first of all, that, as it follows from Definition 3 and relation (6), 
for an arbitrary value $T_{1}, 0<T_{1}<1$, there is a number $N=N\left(T_{1}\right)$ such that the relation

$$
\alpha_{\left.J_{\langle} n\right\rangle}(t)<T_{1} \text { for all } n \geq N
$$

is valid at any point $t \in I^{\prime}$.

Further, as it follows from (5) and (6), there is a number $T_{2}$ such that the relation

$$
0<\alpha_{j}^{\prime}(t)<\gamma<1, \quad j=1,2,
$$

holds at all points $t, 0 \leq t \leq T_{2}$, for some constant $\gamma$. Take an arbitrary number $T_{1}<1$ and let $T=\min \left(T_{1}, T_{2}\right)$. Now introduce a number $N>1$ such that at all points $t>0$ and for $n \geq N$ the relations

$$
\alpha_{J_{\langle n\rangle}}(t)<T \quad \text { and, consequently, } \quad \alpha_{j}^{\prime}\left(\alpha_{J_{\langle n\rangle}}(t)\right)<\gamma
$$

are valid. Let us prove the differentiability of the function $F$. Consider the series $\sum_{J}\left|H^{\prime}\left(\alpha_{J}(t)\right) \alpha_{J}^{\prime}(t)\right|$ and write down it in the form

$$
\sum_{n=0}^{N-1} \sum_{J_{\langle n\rangle}}\left|H^{\prime}\left(\alpha_{J_{\langle n\rangle}}(t)\right) \alpha_{J_{\langle n\rangle}}^{\prime}(t)\right|+\sum_{n=N}^{\infty} \sum_{J_{\langle n\rangle}}\left|H^{\prime}\left(\alpha_{J_{\langle n\rangle}}(t)\right) \alpha_{J_{\langle n\rangle}}^{\prime}(t)\right|,
$$

with $\sum_{J_{\langle n\rangle}}$ being the summation over all multiindices $J_{\langle n\rangle}=\left(j_{1}, \ldots, j_{n}\right), n=$ $1,2, \ldots$. If we use condition (3) and the inequality $\left|H^{\prime}(t)\right|<c t$ with $c$ a positive constant, which follows (3), then we conclude that the second term in (19) is majorized by the function

$$
\sum_{m=1}^{\infty} A_{m}(t)=\sum_{m=1}^{\infty} \sum_{J_{\langle m\rangle}} \sum_{J_{\langle N\rangle}} \alpha_{J_{\langle m\rangle}}\left(\alpha_{J_{\langle N\rangle}}(t)\right)\left|\left(\alpha_{J_{\langle m\rangle}}\left(\alpha_{J_{\langle N\rangle}}\right)\right)^{\prime}\right| .
$$

We are now going to estimate from above the function $A_{m}(t), m=1,2$. Taking into account the positiveness of the functions $\alpha_{1}(t)$ and $\alpha_{2}(t)$ for $t>0$ we find that

$$
\begin{aligned}
A_{m}(t) & =\sum_{J_{\langle m-1\rangle}} \sum_{J_{\langle N\rangle}} \alpha_{1} \circ \alpha_{J_{\langle m-1\rangle}}\left(\alpha_{J_{\langle N\rangle}}(t)\right)\left|\left(\alpha_{1} \circ \alpha_{J_{\langle m-1\rangle}}\left(\alpha_{J_{\langle N\rangle}}(t)\right)\right)^{\prime}\right| \\
& +\sum_{J_{\langle m-1\rangle}} \sum_{J_{\langle N\rangle}} \alpha_{2} \circ \alpha_{J_{\langle m-1\rangle}}\left(\alpha_{J_{\langle N\rangle}}(t)\right)\left|\left(\alpha_{2} \circ \alpha_{J_{\langle m-1\rangle}}\left(\alpha_{J_{\langle N\rangle}}(t)\right)\right)^{\prime}\right| \\
= & \sum_{J_{\langle m-1\rangle}} \sum_{J_{\langle N\rangle}}\left(\alpha_{1}\left(\alpha_{J_{\langle m-1\rangle}}\left(\alpha_{\left.J_{\langle N\rangle}\right\rangle}\right)\right) \alpha_{1}^{\prime}\left(\alpha_{J_{\langle m-1\rangle}}\left(\alpha_{J_{\langle N\rangle}}\right)\right)\right)\left|\left(\alpha_{J_{\langle m-1\rangle}}\left(\alpha_{J_{\langle N\rangle}}\right)\right)^{\prime}\right| \\
+ & \sum_{J_{\langle m-1\rangle}} \sum_{J_{\langle N\rangle}}\left(\alpha_{2}\left(\alpha_{J_{\langle m-1\rangle}}\left(\alpha_{J_{\langle N\rangle}}\right)\right) \alpha_{2}^{\prime}\left(\alpha_{J_{\langle m-1\rangle}}\left(\alpha_{J_{\langle N\rangle}}\right)\right)\right)\left|\left(\alpha_{J_{\langle m-1\rangle}}\left(\alpha_{J_{\langle N\rangle}}\right)\right)^{\prime}\right| \\
\leq \gamma & \sum_{J_{\langle m-1\rangle}} \sum_{J_{\langle N\rangle}} \alpha_{J_{\langle m-1\rangle}}\left(\alpha_{J_{\langle N\rangle}}\right)\left|\left(\alpha_{J_{\langle m-1\rangle}}\left(\alpha_{J_{\langle N\rangle}}\right)\right)^{\prime}\right|=\gamma A_{m-1}(t)
\end{aligned}
$$

On the last step the relations (18) and (5) were used. This proves the uniform convergence of the series (19) and, at the same time, the differentiability of the function $F(t)$. 
It remains to prove that $F \in C^{2}\left(I^{\prime}\right)$, if $H \in C^{2}\left(I^{\prime}\right)$. To do this, it suffices as above, to establish the uniform convergence of the series

$$
\begin{aligned}
\sum_{n=N}^{\infty} \sum_{J_{\langle n\rangle}}\left(H\left(\alpha_{J_{\langle N\rangle}}(t)\right)\right)^{\prime \prime} & =\sum_{n=N}^{\infty} \sum_{J_{\langle n\rangle}} H^{\prime \prime}\left(\alpha_{J_{\langle n\rangle}}(t)\right)\left(\alpha_{J_{\langle n\rangle}}^{\prime}(t)\right)^{2} \\
& +\sum_{n=N}^{\infty} \sum_{J_{\langle n\rangle}} H^{\prime}\left(\alpha_{J_{\langle n\rangle}}(t)\right) \alpha_{J_{\langle n\rangle}^{\prime \prime}}^{\prime \prime}(t):=\sum_{1}+\sum_{2},
\end{aligned}
$$

where the relation

$$
(f(g))^{\prime \prime}=f^{\prime \prime}(g) g^{\prime 2}+f^{\prime}(g) g^{\prime \prime}
$$

has been used. To prove the convergence of the series $\sum_{1}$ consider, as above, the series $\sum_{m=1}^{\infty} \mathcal{B}_{m}(t)$, majorizing $\sum_{1}$, where

$$
\mathcal{B}_{m}(t)=\sum_{J_{\langle m\rangle}} \sum_{J_{\langle N\rangle}}\left(\alpha_{J_{\langle m\rangle}}\left(\alpha_{J_{\langle N\rangle}}(t)\right)\right)^{\prime^{2}}
$$

Having used again the relation $\alpha_{J_{\langle N\rangle}}(t) \in(0, T)$ for all points $t>0$, we find that $\alpha_{j}^{\prime}\left(\alpha_{J_{\langle N\rangle}}(t)\right) \leq \gamma$, and hence

$$
\begin{aligned}
\mathcal{B}_{m}(t) & =\sum_{J_{\langle m-1\rangle}} \sum_{J_{\langle N\rangle}}\left(\alpha_{1}\left(\alpha_{J_{\langle m-1\rangle}}\left(\alpha_{J_{\langle N\rangle}}(t)\right)\right)\right)^{\prime^{2}}+\left(\alpha_{2}\left(\alpha_{J_{\langle m-1\rangle}}\left(\alpha_{J_{\langle N\rangle}}(t)\right)\right)\right)^{\prime^{2}} \\
& =\sum_{J_{\langle m-1\rangle}} \sum_{J_{\langle N\rangle}}\left(\alpha_{1}^{\prime}\left(\alpha_{J_{\langle m-1\rangle}}\left(\alpha_{J_{\langle N\rangle}}(t)\right)\right)\right)^{2}\left(\alpha_{J_{\langle m-1\rangle}}\left(\alpha_{J_{\langle N\rangle}}(t)\right)\right)^{\prime^{2}} \\
& +\left(\alpha_{1}^{\prime}\left(\alpha_{J_{\langle m-1\rangle}}\left(\alpha_{J_{\langle N\rangle}}(t)\right)\right)\right)^{2}\left(\alpha_{J_{\langle m-1\rangle}}\left(\alpha_{J_{\langle N\rangle}}(t)\right)\right)^{\prime^{2}} \leq \gamma^{2} \mathcal{B}_{m-1}(t)
\end{aligned}
$$

This proves the convergence of the series $\sum_{1}$. It remains to establish the uniform convergence of the series $\sum_{2}$, or, remembering that $\left|H^{\prime}(t)\right| \leq c t$, the analogous convergence of the series $\sum_{m=1}^{\infty} \mathcal{C}_{m}(t)$, where

$$
\mathcal{C}_{m}(t)=\sum_{J_{\langle m\rangle}} \sum_{J_{\langle N\rangle}} \alpha_{J_{\langle m\rangle}}\left(\alpha_{J_{\langle N\rangle}}(t)\right)\left|\left(\alpha_{J_{\langle m\rangle}}\left(\alpha_{J_{\langle N\rangle}}(t)\right)\right)^{\prime \prime}\right| .
$$

Using, as above, relation (20) and the identity $\alpha_{J_{\langle m\rangle}}=\alpha_{1} \circ \alpha_{J_{\langle m-1\rangle}}+\alpha_{2} \circ \alpha_{J_{\langle m-1\rangle}}$, we arrive at the inequality 


$$
\begin{aligned}
\mathcal{C}_{m}(t) & =\sum_{J_{\langle m-1\rangle}, J_{\langle N\rangle}} \alpha_{1}\left(\alpha_{J_{\langle m-1\rangle}}\left(\alpha_{J_{\langle N\rangle}}\right)\right)\left|\left(\alpha_{1}\left(\alpha_{J_{\langle m-1\rangle}}\left(\alpha_{J_{\langle N\rangle}}\right)\right)\right)^{\prime \prime}\right| \\
& +\sum_{J_{\langle m-1\rangle}, J_{\langle N\rangle}} \alpha_{2}\left(\alpha_{\left.J_{\langle m-1\rangle}\right\rangle}\left(\alpha_{J_{\langle N\rangle}}\right)\right)\left|\left(\alpha_{2}\left(\alpha_{J_{\langle m-1\rangle}}\left(\alpha_{\left.J_{\langle N\rangle}\right\rangle}\right)\right)\right)^{\prime \prime}\right| \\
\leq & \sum_{J_{\langle m-1\rangle}, J_{\langle N\rangle}}\left(\alpha_{1}\left(\alpha_{J_{\langle m-1\rangle}}\left(\alpha_{J_{\langle N\rangle}}\right)\right)\left|\alpha_{1}^{\prime \prime}\left(\alpha_{J_{\langle m-1\rangle}}\left(\alpha_{J_{\langle N\rangle}}\right)\right)\right|\left(\alpha_{J_{\langle m-1\rangle}}\left(\alpha_{J_{\langle N\rangle}}\right)\right)^{\prime 2}\right. \\
& \left.+\alpha_{2}\left(\alpha_{\left.J_{\langle m-1\rangle}\right\rangle}\left(\alpha_{J_{\langle N\rangle}}\right)\right)\left|\alpha_{2}^{\prime \prime}\left(\alpha_{J_{\langle m-1\rangle}}\left(\alpha_{J_{\langle N\rangle}}\right)\right)\right|\left(\alpha_{J_{\langle m-1\rangle}}\left(\alpha_{\left.J_{\langle N\rangle}\right\rangle}\right)\right)^{\prime 2}\right) \\
& +\sum_{J_{\langle m-1\rangle}, J_{\langle N\rangle}}\left(\alpha _ { 1 } ( \alpha _ { J _ { \langle m - 1 \rangle } } ( \alpha _ { J _ { \langle N \rangle } \rangle } ) ) \alpha _ { 1 } ^ { \prime } \left(\alpha_{J_{\langle m-1\rangle}}\left(\alpha_{\left.J_{\langle N\rangle}\right)}\right)\left|\left(\alpha_{J_{\langle m-1\rangle}}\left(\alpha_{J_{\langle N\rangle}}\right)\right)^{\prime \prime}\right|\right.\right. \\
& \left.+\alpha_{2}\left(\alpha_{\left.J_{\langle m-1\rangle}\right\rangle}\left(\alpha_{J_{\langle N\rangle}}\right)\right) \alpha_{2}^{\prime \prime}\left(\alpha_{J_{\langle m-1\rangle}}\left(\alpha_{J_{\langle N\rangle}}\right)\right)\left|\left(\alpha_{J_{\langle m-1\rangle}}\left(\alpha_{\left.J_{\langle N\rangle}\right\rangle}\right)\right)^{\prime \prime}\right|\right) \\
\leq & c \sum_{J_{\langle m-1\rangle}, J_{\langle N\rangle}}\left(\alpha_{J_{\langle m-1\rangle}}\left(\alpha_{\left.J_{\langle N\rangle}\right\rangle}\right)\right)^{{ }^{2}} \\
& +\gamma \sum_{J_{\langle m-1\rangle}, J_{\langle N\rangle}} \alpha_{J_{\langle m-1\rangle}}\left(\alpha_{J_{\langle N\rangle}}\right)\left|\left(\alpha_{J_{\langle m-1\rangle}}\left(\alpha_{J_{\langle N\rangle}}\right)\right)^{\prime \prime}\right|=c \mathcal{B}_{m-1}(t)+\gamma \mathcal{C}_{m-1}(t),
\end{aligned}
$$

where $c=\max \left\{\sup \mid \alpha_{1}^{\prime \prime}(t)\right.$, sup $\left.\left|\alpha_{2}^{\prime \prime}(t)\right|\right\}$. From what was proved above it follows that the series $\sum_{m=1}^{\infty} \mathcal{B}_{m}(t)$ converges uniformly, and, therefore, it is true with respect to the series $\sum_{m=1}^{\infty} \mathcal{C}_{m}(t)$. This completes the proof of Theorem 4 for $k=2$.

\subsection{Proof of Theorem 5 .}

Note first of all that without loss of generality we can restrict ourselves to the case of equation

$$
F(t)-F\left(\delta_{1}(t)\right)-F\left(\delta_{2}(t)\right)=0, \quad t \in I,
$$

where

$$
\delta_{1}^{\prime}(t)+\delta_{2}^{\prime}(t)=1, \quad t \in I, \quad \text { and } \quad \delta_{1}^{\prime}(-1) \delta_{2}^{\prime}(1)>0 .
$$

Indeed, by the first relation in (12) the map $\alpha(t)=\alpha_{1}(t)+\alpha_{2}(t)$ from $I$ into itself is invertible. In addition, the boundary $\partial I$ is invariant with respect to this map. The change of variable $\alpha(t) \rightarrow t$ reduces equation (14) to equation (21) with

$$
\delta_{1}=\alpha_{1} \circ \alpha^{-1}, \quad \delta_{2}=\alpha_{2} \circ \alpha^{-1} .
$$

The new guiding sets $\mathcal{T}_{j}=\left\{t \mid \delta_{j}^{\prime}(t)=1\right\}, j=1,2$, are nothing but the sets $\alpha^{-1} \mathcal{T}_{j}$ with the "old" $\mathcal{T}_{j}$. The second relation in (12) and relation (13) remain true in the new coordinate system due to relation $\alpha^{\prime}(t)>0$. In order to avoid some technical details we consider the only case where the set $\mathcal{T}_{2}$ is finite. The general case can be studied on the basis of some specific results related to the attractors of dynamical systems in question (see [P7]).

Introduce the notation

$$
(\mathcal{B} F)(t)=F(t)-F\left(\delta_{1}(t)\right)-F\left(\delta_{2}(t)\right) .
$$

Substituting successively the values $t=-1$ and $t=1$ in the relation $(\mathcal{B} F)(t)=0$ we find, remembering the definition of the function $\delta_{j}(t)$, that

$$
(\mathcal{B F})(0)=0 .
$$


This makes it possible to conclude that, for an arbitrary function $F(t) \in C_{1}(I)$, relation (21) is equivalent to the relation

$$
F^{\prime}(t)-\delta_{1}^{\prime}(t) F^{\prime}\left(\delta_{1}(t)\right)-\delta_{2}^{\prime}(t) F^{\prime}\left(\delta_{2}(t)\right)=0, \quad t \in I .
$$

To simplify notation let

$$
a_{j}(t)=\delta_{j}^{\prime}(t), \quad j=1,2, \quad \text { and } \quad F^{\prime}=G .
$$

Then equation (23) becomes

$$
G(t)-a_{1}(t) G\left(\delta_{1}(t)\right)-a_{2}(t) G\left(\delta_{2}(t)\right)=0, \quad t \in I,
$$

where, by (22),

$$
\begin{gathered}
a_{1}(t)+a_{2}(t)=1 \quad a_{j}(t) \geq 0, \quad j=1,2 ; \quad t \in I, \\
a_{1}(-1) a_{2}(1) \neq 0,
\end{gathered}
$$

and

$$
\mathcal{T}_{j}=\left\{t \mid a_{j}(t)=1\right\}, \quad j=1,2 .
$$

To prove Theorem 5 it remains to show that the only solution of equation (24) is an arbitrary constant $F(t)=C$. We will do this in several steps.

I. Let us show that the extremal values of any solution $G(t) \in C(I)$ of equation (24) spread only along $\mathcal{T}$ - proper orbits.

To this end, let $M=\max _{I} G$ and let $\mathfrak{M}=\{t \in I \mid F(t)=M\}$. If $G(\widehat{t})=M$, then $G\left(\delta_{1}(\widehat{t})\right)=M-\varepsilon_{1}, G\left(\delta_{2}(\widehat{t})\right)=M-\varepsilon_{2}$ for some nonnegative numbers $\varepsilon_{1}$ and $\varepsilon_{2}$. Substituting $\widehat{t}$ for $t$ in equation (24) we find that $a_{1}(\widehat{t}) \varepsilon_{1}+a_{2}(\widehat{t}) \varepsilon_{2}=0$. It follows that if $\widehat{t} \in \mathfrak{M} \backslash \mathcal{T}$, then $\varepsilon_{1}=\varepsilon_{2}=0$, and hence $\delta_{1}(\widehat{t}) \in \mathfrak{M}, \delta_{2}(\widehat{t}) \in \mathfrak{M}$. But if $\widehat{t} \in \mathfrak{M} \cap \mathcal{T}_{1}$, then $\varepsilon_{1}=0$, and consequently $\delta_{1}(\widehat{t}) \in \mathfrak{M}$. In just the same way, if $\widehat{t} \in \mathfrak{M} \cap \mathcal{T}_{2}$, then $\delta_{2}(\widehat{t}) \in \mathfrak{M}$. Combining these observations with the definition of $\mathcal{T}$ - proper orbit we conclude that together with every point $t_{1} \in \mathfrak{M}$ the next point of any $\mathcal{T}$ - proper orbit $\left(t_{1}, t_{2}\right)$ also belongs to $\mathfrak{M}$. As $\delta_{1}$ and $\delta_{2}$ are maps in $I$, this argument can be applied to the point $t_{2}$. As a result we obtain one or two points $t_{3}$ of the form $t_{3}=\delta_{j_{2}}\left(t_{2}\right)=\delta_{j_{2}} \circ \delta_{j_{1}}\left(t_{1}\right)$ lying in the set $\mathfrak{M}$, so that the orbit $\mathcal{O}=\left(t_{1}, t_{2}, t_{3}\right)$ turns out to be $\mathcal{T}$ - proper. Arguing in the same way, we conclude that together with every point $t_{1} \in \mathfrak{M}$ any its $\mathcal{T}$ - proper orbit $\mathcal{O}=\left(t_{1}, t_{2}, \ldots\right)$ is situated in the set $\mathfrak{M}$. This proves the above assertion with respect to the maximal value of the function $G(t)$. But what has been said above with respect to spreading maximal value $M$ of a solution $F$ along $\mathcal{T}$ - proper orbits remains valid for a minimal value $m$ of the same solution. This completes the proof of assertion.

II. Assume that for an arbitrary point $t \in I$ there is a $\mathcal{T}$-proper orbit $\left(t, t_{1}\right.$, $\left.t_{2}, \ldots\right)$ converging to one of the boundary points of $I$. Then, by continuity of the function $G(t)$, it attains its extremal values at the points $t=-1$ and $t=1$. Substituting consecutively these points for $t$ in equation (24) and using relations (2), (25) and (26) we arrive at the relation

$$
G(-1)=G(1)=G(0) .
$$

This means that the maximal and minimal values of $G(t)$ coincide, and hence, $G(t)$ is a constant. But this immediately implies the relation $F(t)=\lambda t$ and thus completes the proof of Theorem 5 .

III. We now pass to the main technical part of the proof of Theorem 5: the proof of the assertion formulated at the beginning of II. 
Lemma 7 If $t \neq 0$ and $\left(t_{1}, t\right),\left(t_{2}, t\right)$ are two $\mathcal{T}$ - proper orbits, then $t_{1}=t_{2}$.

Proof. If $t_{1} \neq t_{2}$ and

$$
t=\delta_{j_{1}}\left(t_{1}\right), \quad t=\delta_{j_{2}}\left(t_{2}\right),
$$

then $j_{1}=j_{2}$, as the ranges of the maps $\delta_{1}$ and $\delta_{2}$ have the only common point $t=0$. By the monotonicity properties of the maps $\delta_{j}$, it is possible only if $\delta_{j_{1}}(t)=$ const at all points $t$ between $t_{1}$ and $t_{2}$. But then $\delta_{j_{1}}^{\prime}(t) \equiv 0$ for these $t$, and, in particular, $\delta_{j_{1}}^{\prime}\left(t_{1}\right)=0$. However, this contradicts to the definition of $\mathcal{T}$ - proper orbit: according this definition only the orbit $\left(t_{1}, \delta_{j^{\prime}}\left(t_{1}\right)\right)$ with $j^{\prime} \neq j_{1}$ is $\mathcal{T}$ - proper.

Remark From a geometrical point of view this fact means that two different $\mathcal{T}$ proper orbits cannot enter at the same point $t \in I \backslash\{0\}$.

Lemma 8 If a cyclic orbit $\Sigma$ is a part of a $\mathcal{T}$ - proper orbit $\mathcal{O}=\left(t_{1}, t_{2}, \ldots\right)$, then $t_{1} \neq 0$ and $t_{1} \in \Sigma$.

Proof. If $t_{1} \notin \Sigma$ we let $t_{q}, q \geq 2$, be the first point in $\mathcal{O}$, situated in $\Sigma$, so that

$$
\Sigma=\left(t_{q}, t_{q+1}, \ldots, t_{q+n}\right),
$$

and $t_{q}=t_{q+n}$. But then $t_{q-1} \neq t_{q+n+1}$ as $t_{q-1} \notin \Sigma$ and $t_{q+n+1} \in \Sigma$, and, by Lemma 7 , for none of indices $j_{1}, j_{2}$ the relation

$$
t_{q}=\delta_{j_{1}}\left(t_{q-1}\right)=\delta_{j_{2}}\left(t_{q+n-1}\right)
$$

is possible. This proves that $t_{1} \in \Sigma$.

To complete the proof of the lemma it remains to note that the relation $t_{1}=0$ is impossible due to (2): only the points $t=-1$ and $t=1$ can precede the point $t_{1}=0$, but they both have no predecessors on a cyclic orbit.

We now note that, if both sets $\mathcal{T}_{1}$ and $\mathcal{T}_{2}$ are infinite, then, by (13), for a sufficiently small $\varepsilon>0$, there is a neighborhood $U_{1}=(-1,-1+\varepsilon)$ such that $\delta_{2}^{\prime}(t)>0$ for all points $t \in U_{1}$. It follows that for any such $t$ the orbit $\mathcal{O}=$ $\left(\delta_{2}(t), \delta_{2}^{2}(t), \ldots, \delta_{2}^{k}(t), \ldots\right)$ is $\mathcal{T}$ - proper and converges to the point $t=-1$. In just the same way, there is a neighborhood $U_{2}=(1-\varepsilon, 1)$ such that the orbit $\mathcal{O}_{1}=\left(t, \delta_{1}(t), \delta_{1}^{2}(t), \ldots\right)$, remaining $\mathcal{T}$ - proper, converges to the point $t=1$ for an arbitrary $t \in U_{2}$.

However, if one of the sets, say $\mathcal{T}_{2}$, is finite, than this property is guaranteed only for the neighborhood $U_{2}$.

Lemma 9 If the above $\varepsilon>0$ is sufficiently small then there is an integer $\nu$ such that

$$
\delta_{1}^{\nu}(t)>1-\varepsilon \quad \text { and } \quad \delta_{2}^{\nu}(t)<-1+\varepsilon
$$

for all points $t \in I$.

Proof. To begin with, we note that the relation

$$
\delta_{2}(t)<t<\delta_{1}(t), \quad t \neq \pm 1,
$$

holds. For example, the second relation is trivial for $t \leq 0$, by Definition 2. If $\delta_{1}(\widehat{t})=\widehat{t}$ for some value $\widehat{t}, 0<\widehat{t}<1$, then by $(22), \delta_{2}(\widehat{\widehat{t}})=0$, and by the same Definition $2, \delta_{2}(t) \equiv 0$ on the interval $(\widehat{t}, 1)$. But then $\delta_{2}^{\prime}(1)=0$, what contradicts to the second relation (12). This proves the inequality $t<\delta_{1}(t)$ for all $t \neq 1$. In turn, the latter inequality implies the relation $\delta_{1}^{\nu}(-1) \rightarrow 1$, as $\nu \rightarrow \infty$. Indeed, the sequence $\delta_{1}(-1), \delta_{1}^{2}(-1), \ldots$ is bounded and increases. If $\lim \delta_{1}^{\nu}(-1)=\xi$, then, by continuity of $\delta_{1}, \lim \delta_{1}^{\nu+1}(-1)=\delta_{1}(\xi)$, whence $\delta_{1}(\xi)=\xi$, and $\xi=1$. In the same way $\delta_{2}^{\nu}(1) \rightarrow-1$, as $\nu \rightarrow \infty$. This proves the lemma for the points $t=1$ and $t=-1$. The case of an arbitrary $t$ follows now by condition $1^{\circ}$ in Definition 2 . This completes the proof of Lemma 9. 
Corollary 10 If the last point of some $\mathcal{T}$ - proper orbit $\mathcal{O}\left(t_{1}, t_{2}, \ldots, t_{N}\right)$ lies in the set $U_{1}\left(U_{2}\right.$ resp.) (for some $\left.\varepsilon>0\right)$, then the extended orbit $\mathcal{O}^{\prime}=\left(t_{1}, \ldots, t_{N}, \delta_{1}\left(t_{N}\right)\right.$, $\left.\delta_{1}^{2}\left(t_{N}\right), \ldots\right)\left(\mathcal{O}^{\prime}=\left(t_{1}, \ldots, t_{N}, \delta_{2}\left(t_{N}\right), \delta_{2}^{2}\left(t_{N}\right), \ldots\right)\right.$, resp. $)$ converges to the point $t=-1(t=1$, resp. $)$

The proof is evident.

Now everything is ready to complete the proof of Theorem 5 . Let $M$ be the number of points of the set $\mathcal{T}_{2}$. Then, given an arbitrary point $t_{1} \notin \mathcal{T}_{2}$, we create the $\mathcal{T}$ - proper orbit $\mathcal{O}_{1}=\left(t_{1}, \delta_{1}\left(t_{1}\right), \ldots, \delta_{1}^{N}\left(t_{1}\right)\right)$ which moves toward the point $t=1$. If $N=\nu$, then the last point of $\mathcal{O}_{1}$ lies in $U_{2}$ and, by Corollary 10, Theorem 5 is proved. If for some $N<\nu$ the point $t_{2}=\delta_{1}^{N}\left(t_{1}\right)$ lies in $\mathcal{T}_{2}$, then we create the $\mathcal{T}$ - proper orbit $\left(t_{2}, \delta_{2}\left(t_{2}\right), \delta_{2}^{2}\left(t_{2}\right), \ldots, \delta_{2}^{k}\left(t_{2}\right)\right)$ moving toward $t=-1$. As above, if $k=\nu$, then the proof of Theorem 5 is finished by using the extended orbit, due to Corollary 10. If $k<\nu$ and $\delta_{2}^{k}\left(t_{2}\right) \in \mathcal{T}_{1}$, we extend the sewed orbit $\left(t_{1}, \ldots, \delta_{1}^{N}\left(t_{1}\right), \delta_{2} \circ\right.$ $\left.\delta_{1}^{N}\left(t_{1}\right), \ldots, \delta_{2}^{k} \circ \delta_{1}^{N}\left(t_{1}\right)\right)$ by the points $\delta_{1} \circ \delta_{2}^{k}\left(t_{2}\right), \ldots, \delta_{1}^{N_{1}} \circ \delta_{2}^{k}\left(t_{2}\right)$ and so on. As it follows from Lemma 8 , following this procedure we will never return at one of the previous points $t_{j}$ of the orbit in question, with the exception, may be, of the point $t_{1}$. It is clear, that if we do not meet the point $t_{1}$ after $2 M \nu$ described steps, the corresponding point $t_{2 M \nu+1}$ turns out to be in the set $U_{1} \cup U_{2}$. After thisj it remains to sew the orbit $\left(t_{1}, \ldots, t_{2 M \nu+1}\right)$ with the mentioned orbit $\left(\delta_{j}\left(t_{2 M \nu+1}\right), \delta_{j}^{2}\left(t_{2 M \nu+1}\right), \ldots\right)$ with $j=1$ or 2 .

Consider the concluding situation: moving along the orbit $\mathcal{O}$ we achieved the point $t_{1}$. This means that we deal with a periodic $\mathcal{T}$ - proper orbit $\Sigma=\left(t_{1}, \ldots, t_{m}\right)$ which is a suborbit of a $\mathcal{T}$ - proper orbit $\mathcal{O}$ we construct. But (attention!), by the crucial hypothesis $\mathfrak{N}_{\delta}^{\mathcal{T}}=\emptyset$, this cycle $\Sigma$ is not $\mathcal{T}$ - guided. Consequently, $\Sigma$ contains a point $t_{q}, 1 \leq q \leq m-1$, which does not belong to the guiding set $\mathcal{T}$. If, when constructing the orbit $\mathcal{O}$, we left the point $t_{q}$ by means of a map $\delta_{j}$ (i.e. $t_{q+1}=\delta_{j}\left(t_{q}\right)$ ), then now we introduce the new point $\widehat{t}_{q+1}=\delta_{j^{\prime}}\left(t_{k}\right), j^{\prime} \neq j$. Extending the orbit $\mathcal{O}=\left(t_{1}, \ldots, t_{q}, \widehat{t}_{q+1}\right)$ as above, we arrive at a new $\mathcal{T}$-proper orbit $\widetilde{O}=\left(t_{1}, \ldots, t_{q}, \widehat{t}_{q+1}, \widehat{t}_{q+2}, \ldots\right)$. But this time it can't meet one of its previous points (by Lemma 8) and, consequently, after not more than $2 M \nu$ steps our $\mathcal{T}$ proper orbit enters in the neighborhood $U_{1} \cup U_{2}$. To complete the proof of Theorem 5 it remains to use Corollary 10.

Remark If the point $t_{1}$ (see the text straight away after Corollary 10) lies in $\mathcal{T}_{2}$, then we consider the point $t_{1}^{*}=\delta_{2}\left(t_{1}\right)$ and repeat all the above arguments in connection with this point.

\subsection{Proof of Theorem 6.}

As in the proof of Theorem 5 we reduce problem (10) to the equivalent problem

$$
\mathcal{B} F(t):=F(t)-F\left(\delta_{1}(t)\right)-F\left(\delta_{2}(t)\right)=h(t) \quad \text { on } I, \quad F(1)=\lambda
$$

with $\delta_{1}(t)$ and $\delta_{2}(t)$ satisfying hypothesis $(22)$. By the invariance of the boundary $\partial I$ with respect to the transformation $t \rightarrow \alpha(t)$, the hypotheses (8) and (9) become the necessary conditions for solvability of problem (28) having form

$$
\mathcal{B} F(-1)=\mathcal{B} F(1)=-F(0)
$$

and

$$
h(-1)=h(1)
$$


Replacing $F(t)$ by $F(t)+t(\lambda+h(1))-h(1)$ in (28) we arrive at the problem

$$
\mathcal{B} F=\widehat{h} \quad \text { on } I, F(0)=0, F(1)=0,
$$

where $\widehat{h}(t)=h(t)-h(1)$. This form is precisely starting point in studying problem (10). As is easily seen, both functions $\mathcal{B} F(t)$ and $\widehat{h}(t)$ vanish at the points $t=-1$ and $t=1$. Therefore, differentiating successively the equation $B F(t)=\widehat{h}_{\mu}(t)$, we obtain two new problems which are equivalent to the problem (29). These new problems are

$$
\begin{gathered}
B_{1} F^{\prime}(t):=F^{\prime}(t)-\delta_{1}^{\prime}(t) F^{\prime}\left(\delta_{1}(t)\right)-\delta_{2}^{\prime}(t) F^{\prime}\left(\delta_{2}(t)\right)=\widehat{h}_{\mu}^{\prime}(t), \\
B_{2} F^{\prime \prime}(t):=F^{\prime \prime}(t)-{\delta_{1}^{\prime}}^{2}(t) F^{\prime \prime}\left(\delta_{1}(t)\right)-{\delta_{2}^{\prime}}^{2}(t) F^{\prime \prime}\left(\delta_{2}(t)\right)-K F^{\prime \prime}(t)=\widehat{h}_{\mu}^{\prime \prime}(t),
\end{gathered}
$$

where $F^{\prime}(t)$ and $F^{\prime \prime}(t)$ stand for the correspondent derivatives, and $K$ denotes a linear operator in $C(I)$

$$
K: H(t) \mapsto \delta_{1}^{\prime \prime}(t) \int_{\xi}^{\delta_{1}(t)} H(s) d s+\delta_{2}^{\prime \prime}(t) \int_{\xi}^{\delta_{2}(t)} H(s) d s .
$$

Here $\xi$ is an arbitrary point of the interval $(0,1)$ such that $F^{\prime}(\xi)=0$. The existence of such point $\xi$ is guaranteed by the boundary conditions in (29). For the sake of brevity we omit the conditions $F(0)=0, F(1)=0$ in (30) and (31). What is important here is that $K$ is a compact operator in the space $C(I)$. This is easily verified by virtue of the Arzelà criterion.

Since all the three problems (29), (30) and (31) in question are equivalent, the solvability of problem (10) under condition (15) will be proved if we establish the injectivity of the operator $B_{1}$, and also show that the index ind $B_{2}$ of the operator $B_{2}$ in the space $C(I)$ is zero.

To prove the injectivity of the operator $B_{1}$ in the space of functions $F$ vanishing at the points $t=0$ and $t=1$ we set $\widehat{h}_{\mu}^{\prime}=0$ in (30). By relation (25) the new equation coincides with problem (24) and, by Theorem 5, all its solutions $F^{\prime}$ are constants. These constants are nothing except zero, since the function $F$ vanishes at two points. If remains to prove that ind $\mathcal{B}_{2}=0$.

To this end we represent the operator $B_{2}$ in the form $\mathcal{B}_{2}=E-L-K$, where $E$ is the identical operator in the space $C(I)$, and $L$ stands for the linear operator of the form

$$
L: f(t) \rightarrow \delta_{1}^{\prime 2}(t)\left(f \circ \delta_{1}\right)(t)+{\delta_{2}^{\prime}}^{2}(t)\left(f \circ \delta_{2}\right)(t) .
$$

in the same space. Having proved the invertibility of the operator $E-L$ in the space $C(I)$, and then applying the Riesz-Shauder theorem to the operators $(E-L)^{-1}$ and $K$, we arrive at the required relation ind $\mathcal{B}_{2}=0$. The existence of the operator $(E-L)^{-1}$ is an almost evident corollary of the following result.

Lemma 11 Under conditions of Theorem 6 there is a natural number $N$ such that the relation

$$
\left\|L^{N}\right\|<1
$$

holds with $\|\cdot\|$ being the standard norm in the space of linear operators from $C(I)$ into itself.

Remark Under condition (32) the operator $(E-L)^{-1}$ is defined as the sum of the converging series $\sum_{m=0}^{N-1} \sum_{k=0}^{\infty} L^{k N+m}$.

Proof. Introduce the following notation

$$
a_{j}(t)={\delta_{j}^{\prime}}^{2}(t) ; \quad a_{j_{2}}\left(\delta_{j_{1}}(t)\right)=a_{j_{1} j_{2}}(t) ; \quad a_{j_{3}}\left(\delta_{j_{2}} \circ \delta_{j_{1}}(t)\right)=a_{j_{1} j_{2} j_{3}}(t) ; \ldots
$$


with all subindices $j, j_{1}, j_{2}, \ldots$ equal to 1 or 2 . By induction on $N$, it is easily verified that the function $\left(L^{N} F\right)(t)$ can be represented in the form

$$
\left(L^{N} F\right)(t)=\sum_{j_{1}, \ldots, j_{N}=1}^{2} a_{j_{1}}(t) a_{j_{1} j_{2}}(t) \ldots a_{j_{1} \ldots j_{N}}(t) F\left(\delta_{J}(t)\right),
$$

where $J=\left(j_{1}, \ldots, j_{N}\right)$ and $\delta_{J}=\delta_{j_{N}} \circ \ldots \circ \delta_{j_{1}}$. It follows, that for an arbitrary function $F$ with $\|F\|=1$ the inequality

$$
\left.\mid L^{N} F\right)(t) \mid \leq \sum_{j_{1}, \ldots, j_{N}=1}^{2} a_{j_{1}}(t) a_{j_{1} j_{2}}(t) \ldots a_{j_{1} \ldots j_{N}}(t)
$$

holds at each point $t \in I$. Denote by $\Lambda_{N}(t)$ the write-hand side of this relation. We will now prove that, for any fixed point $t \in I$, there is a positive number $N$ and a constant $\gamma<1$ such that the relation

$$
\Lambda_{N}(t) \leq \gamma
$$

holds. If $t \notin \mathcal{T}$, then $a_{1}(t)+a_{2}(t)<1$ by (22), and relation (33) is true for $N=1$. If $t \in \mathcal{T}$, consider a $\mathcal{T}$ - proper orbit $\mathcal{O}=\left(t_{1}, t_{2}, \ldots, t_{N}\right)$ with $t_{1}=t$ such that $t_{N}$ is the first point in $\mathcal{O}$ situated in $I \backslash \mathcal{T}$ and $N$ is the minimal number with this property. This means, in particular, that

$$
t_{2}=\delta_{j_{1}^{\prime}}\left(t_{1}\right), \quad t_{3}=\delta_{j_{2}^{\prime}}\left(t_{2}\right), \ldots, t_{N}=\delta_{j_{N-1}^{\prime}}\left(t_{N-1}\right),
$$

for some fixed indices $j_{1}^{\prime}, \ldots, j_{N-1}^{\prime}$ and, in addition,

$$
a_{j_{1}^{\prime}}\left(t_{1}\right)=1, \quad a_{j_{2}^{\prime}}\left(t_{2}\right)=1, \ldots, \quad a_{j_{N-1}^{\prime}}\left(t_{N-1}\right)=1, \quad \sum_{j_{N}=1}^{2} a_{j_{N}}\left(t_{N}\right)<1
$$

The existence of such orbit has been established when proving Theorem 5 (see III, page 12). It is clear that, for an arbitrary natural $m$, the relation $\Lambda_{m} \leq 1$ is true. To see this, it suffices to represent $\Lambda_{m}$ in the form

$$
\Lambda_{m}=\sum_{j_{1}} a_{j_{1}} \sum_{j_{2}} a_{j_{1} j_{2}} \ldots \sum_{j_{m}} a_{j_{1} \ldots j_{m}}
$$

and to use the relation $a_{1}(t)+a_{2}(t) \leq 1$ for all $t \in I$. Setting

$$
A_{j_{1} \ldots j_{N-1}}(t)=a_{j_{1}}(t) a_{j_{1} j_{2}}(t) \ldots a_{j_{1} \ldots j_{N-1}}(t)
$$

and

$$
\mathcal{B}_{j_{1} \ldots j_{N-1}}(t)=\sum_{j_{N}=1}^{2} a_{j_{1} \ldots j_{N-1} j_{N}}(t),
$$

we can rewrite the function $\Lambda_{N}(t)$ in the form

$$
\Lambda_{N}(t)=\sum_{j_{1}, \ldots, j_{N-1}} A_{j_{1} \ldots j_{N-1}}(t) \mathcal{B}_{j_{1} \ldots j_{N-1}}(t) .
$$

It is evident that if, for some nonnegative numbers $p_{k}$ and $q_{k}$, the relations

$$
\sum_{k=1}^{r} p_{k} q_{k} \leq 1, \quad \sum_{k=1}^{r} p_{k} \leq 1, \quad \text { and } q_{k} \leq 1 \text { for all } k,
$$


hold, and at least one number $q_{k^{\prime}}$ is less than one, then

$$
\sum_{k=1}^{r} p_{k} q_{k}<1 .
$$

To make use this observation with respect to the function $\Lambda_{N}(t)$ note that, by the above,

$$
\sum_{j_{1}, \ldots, j_{N-1}} A_{j_{1} \ldots j_{N-1}}(t) \leq 1
$$

Furthermore, by the definition of the multi-index $\left(j_{1}^{\prime}, \ldots, j_{N}^{\prime}\right)$, and by $(34)$, we find that

$$
\mathcal{B}_{j_{1}^{\prime} \ldots j_{N-1}^{\prime}}(t)=\sum_{j_{N}} a_{j_{1}^{\prime} \ldots j_{N-1}^{\prime} j_{N}}(t)=\sum_{j_{N}} a_{j_{N}}\left(t_{N}\right)<1 .
$$

On the other hand, by virtue the same relation (34),

$$
A_{j_{1}^{\prime} \ldots j_{N-1}^{\prime}}(t)=a_{j_{1}^{\prime}}\left(t_{1}\right) a_{j_{2}^{\prime}}\left(t_{2}\right) \ldots a_{j_{N-1}}^{\prime}\left(t_{N-1}\right)=1
$$

Thus, the function $\Lambda_{N}(t)$ has the same structure as the expression $p_{k} q_{k}$ above and, consequently, the inequality $\Lambda_{N}(t)<1$ holds with a constant $\gamma=\gamma(t)<1$.

Note now that, by virtue of the continuity of all the functions in question, relation (33) holds at all points of some neighborhood $\mathcal{U}$ of the point under consideration for the same number $N$, may be with a larger constant $\gamma<1$. The collection of such neighborhoods forms an open covering of the closed set $I \backslash \mathcal{B}$. Let $\left\{\mathcal{U}_{j}\right\}_{j=1}^{k}$ be a finite subsystem of these neighborhoods, and $N_{j}, \gamma_{j}$ the corresponding constants. Taking $m=\max N_{j}$ and $\gamma=\max \gamma_{j}$ we arrive at the desired inequality $\left\|L^{m}\right\|<1$, and this completes the proof of the solvability of problem (10) in the part "if".

To prove the necessity of the hypothesis $\mathfrak{N}_{\alpha}^{\mathcal{T}}=\emptyset$ we assume that $\mathfrak{N}_{\alpha}^{\mathcal{T}} \neq \emptyset$. Then there is a $\mathcal{T}$ - proper $\mathcal{T}$ - guided periodic orbit $\mathcal{O}=\left(t_{1}, \ldots, t_{n+1}\right)$. If a function $F(t)$ solves problem (28), than the function $G=F^{\prime}$ solves the equation

$$
G(t)=\delta_{1}^{\prime} G\left(\delta_{1}(t)\right)-\delta_{2}^{\prime} G\left(\delta_{2}(t)\right)=H(t),
$$

with $H(t)=\widehat{h}_{\mu}^{\prime}(t)$ (see (30)). By definition, the points $t_{1}, \ldots, t_{n+1}$ satisfy the conditions

$$
t_{k+1}=\delta_{j_{k}}\left(t_{k}\right), \quad \delta_{j_{k}^{\prime}}^{\prime}\left(t_{k}\right)=0 \quad \text { for } j_{k}^{\prime} \neq j_{k}, \quad k=1, \ldots, n, \quad \text { and } t_{n+1}=t_{1} .
$$

Let us substitute $t_{1}$ for $t$ in equation (35). Then one of the numbers $\delta_{j}^{\prime}\left(t_{1}\right)$ is equal to zero, whereas $\delta_{j^{\prime}}^{\prime}\left(t_{1}\right)=1$. Furthermore, $t_{2}=\delta_{j^{\prime}}\left(t_{1}\right)$, by the definition of a $\mathcal{T}$ - proper orbit. This leads to the relation $G\left(t_{1}\right)-G\left(t_{2}\right)=H^{\prime}\left(t_{1}\right)$. Continuing this procedure we arrive at the chain of equalities $G\left(t_{2}\right)-G\left(t_{3}\right)=H^{\prime}\left(t_{2}\right), \ldots, G\left(t_{n}\right)-G\left(t_{1}\right)=$ $H^{\prime}\left(t_{n}\right)$, where the periodicity of the orbit $\mathcal{O}$ is used on the last step. Adding all these relations together, we obtain

$$
\sum_{j=1}^{n} H^{\prime}\left(t_{j}\right)=0 .
$$

Thus, in the presence of a $\mathcal{T}$ - proper $\mathcal{T}$ - guided cycle $\mathcal{O}=\left(t_{1}, \ldots, t_{n}\right)$, this relation is a necessary condition for the solvability of problem (28). Hence, the necessity of the condition $\mathfrak{N}_{\zeta}^{\mathcal{T}}=\emptyset$ is proved. To complete the proof of Theorem 7 it remains to establish the continuity of the inverse operator $\mathcal{B}_{\alpha}^{-1}$. The simplest way to do this is using Banach closed graph theorem combined with solvability of problem (28) already proved, and with an obvious a priory estimate

$$
\left\|\mathcal{B} F, C^{2}(I)\right\| \leq \mathrm{const}\left\|F, C^{2}(I)\right\|,
$$

guaranteing the continuity of the operator $\mathcal{B}$. 


\section{Applications.}

The main goal of this section is to show that the Cauchy type functional equations are of the great interest not only by themselves as an object of analysis, but they arise naturally when solving various problems in such diverse fields as integral and functional equations, integral geometry, boundary problems for partial differential equations etc.

In order not to enlarge extremely the size of this paper some of corresponding results are given with full proofs and the proofs are only outlined for some.

\subsection{Multiplicative Cauchy type functional equation.}

This title was introduced in the paper [P4] as the definition for functional equations of the form

$$
F\left(\alpha_{1}(t)+\alpha_{2}(t)\right)=F\left(\alpha_{1}(t)\right) F\left(\alpha_{2}(t)\right) H(t), \quad t \in I^{\prime},
$$

where $H>0$ is a given function, and the functions $\alpha_{1}(t)$ and $\alpha_{2}(t)$ form one of the above configurations.

Below we prove two theorems giving an exhaustive solution of the solvability problem for this equation in both cases of $Z$ - and $\mathcal{P}$ - configurations.

\subsubsection{The case of a $Z$ - configurations.}

We begin with a very simple but crucial lemma which makes it possible to reduce problem (37) to a standard Cauchy type functional equation.

Lemma 12 If functions $\alpha_{1}(t)$ and $\alpha_{2}(t)$ form a $Z$ - configuration, then any nontrivial continuous solution of equation (37) is positive on $I^{\prime}$.

Proof. Let $F(z) \not \equiv 0$ on $I^{\prime}$ and

$$
z_{0}=\min \{z \mid F(z)=0\} .
$$

If $z_{0} \neq 0$ and $F\left(z_{0}\right)=F\left(\alpha_{1}\left(t_{0}\right)+\alpha_{2}\left(t_{0}\right)\right)$, then, by (37), $F\left(\alpha_{1}\left(t_{0}\right)\right)=0$ or $F\left(\alpha_{2}\left(t_{0}\right)\right)=0$. This means that for some $z_{1}<z_{0}$ with $z_{1}=\alpha_{1}\left(t_{0}\right)$ or $z_{1}=\alpha_{2}\left(t_{0}\right)$ one has $F\left(z_{1}\right)=0$. But this contradicts to the definition of $z_{0}$. It remains to exclude the possibility $F(0)=0$. If $F(0)=0$, then for an arbitrary small $\varepsilon>0$ (satisfying also the relation $\varepsilon H(0)<1)$ there is a point $z \in \mathcal{R}\left(\alpha_{1}+\alpha_{2}\right)$ such that $F(z)=\varepsilon$ and $F\left(z^{\prime}\right)<\varepsilon$ for all $z^{\prime}<z$. If $z=\left(\alpha_{1}+\alpha_{2}\right)\left(t_{0}\right)$, then $F\left(\alpha_{1}\left(t_{0}\right)\right)<\varepsilon$ and $F\left(\alpha_{2}\left(t_{0}\right)\right)<\varepsilon$, as $\alpha_{j}(t)>0$ for $t>0$ and $j=1,2$. By (37), this leads to the impossible relation

$$
\varepsilon \leq \varepsilon^{2} H(0)<\varepsilon,
$$

and this completes the proof of the lemma.

This lemma makes it possible to reduce equation (37), by taking the logarithm of both sides of this equation, to the standard Cauchy type functional equation

$$
G\left(\alpha_{1}(t)+\alpha_{2}(t)\right)=G\left(\alpha_{1}(t)\right)+G\left(\alpha_{2}(t)\right)+\mathcal{H}(t), \quad t \in I^{\prime} .
$$

Denote by $\widetilde{C}_{\langle\kappa\rangle}\left(I^{\prime}\right)$ the space of positive continuous functions $f$ on $I^{\prime}$ such that

$$
\ln f(t) \in C_{\langle\kappa\rangle}\left(I^{\prime}\right)
$$

The following result is a direct corollary of Theorem 2 and Theorem 3 . 
Theorem $131^{\circ}$ Assume that the functions $\alpha_{1}(t)$ and $\alpha_{2}(t)$ satisfy condition (7) and belong to the space $C_{\langle 1\rangle}\left(I^{\prime}\right)$. Then all solutions $F$ of the homogeneous equation

$$
F\left(\alpha_{1}(t)+\alpha_{2}(t)\right)=F\left(\alpha_{1}(t)\right) F\left(\alpha_{2}(t)\right) \text { on } I^{\prime}
$$

belonging to the space $\widetilde{C}_{1}\left(I^{\prime}\right)$, have a form $F(z)=e^{\lambda z}, \lambda \in \mathbb{R}$.

$2^{\circ}$ Assume that both functions $\alpha_{1}(t)$ and $\alpha_{2}(t)$ satisfy condition of Theorem 3 . Then for an arbitrary function $H \in \widetilde{C}_{\langle 1+r\rangle}\left(I^{\prime}\right), 0<r<1$, satisfying condition (3), and for any positive number $\lambda$, there is a unique solution $F \in \widetilde{C}_{\langle 1+r\rangle}\left(I^{\prime}\right)$ of the problem

$$
\left.F\left(\alpha_{1}+\alpha_{2}\right)(t)\right)=F\left(\alpha_{1}(t)\right) F\left(\alpha_{2}(t)\right) H(t), \quad \text { on } I^{\prime} ; \quad F(1)=\lambda .
$$

This solution can be represented in the form

$$
F(z)=e^{\mu z} \prod_{J} \mathcal{H}\left(\delta_{J}(z)\right), \quad z \in \widehat{I},
$$

with some (uniquely defined) real $\mu$ and $\delta_{J}$ running all elements of the semigroup $\Phi_{\delta}$, generated by the maps $\delta_{j}=\alpha_{j} \circ \alpha^{-1}, j=1,2$, from the interval $\widehat{I}$ into itself.

\subsubsection{The case of a $\mathcal{P}$ - configuration.}

Let functions $\alpha_{1}(t)$ and $\alpha_{2}(t)$ form a $\mathcal{P}$ - configuration. Then a new point, as compared with the case of a $Z$-configuration, is that solutions of equation (37) are not necessarily strictly positive on the interval $I$, as follows from the

Lemma 14 The equation

$$
F(t)=F\left(\frac{t-1}{2}\right) F\left(\frac{t+1}{2}\right), \quad t \in I,
$$

has infinite set of oscillating solution, i.e. those taking values of opposite signs. ${ }^{1}$

Proof. Note first of all that the functions $\alpha_{1}=(t-1) / 2$ and $\alpha_{2}=(t+1) / 2$ form a $\mathcal{P}$ - configuration on $I$ and satisfy condition (5). As is directly verified, any function

$$
F_{k}(t)=2 \cos (k+1 / 2) \pi t, \quad k=0,1, \ldots
$$

solves equation (40) and oscillates on $I$.

By this lemma, the hypothesis of positiveness of a solution to equation (37) is necessary in the following assertions.

Theorem $151^{\circ}$ Assume that the functions $\alpha_{1}(t)$ and $\alpha_{2}(t)$ belong to the space $C^{1}(I)$ and satisfy conditions (12), (13) and (15). Then all positive $C^{1}$ - solutions to the homogeneous equation (38) are of the form

$$
F(z)=e^{\lambda z}, \lambda \in \mathbb{R} .
$$

$2^{\circ}$ Let the functions $\alpha_{1}(t)$ and $\alpha_{2}(t)$ belong to the space $C^{2}(I)$ and satisfy the same hypotheses as in $1^{\circ}$. Then, for an arbitrary positive function $H \in C^{2}(I)$, satisfying hypothesis (6), and for any real number $\lambda$, there is a unique positive solution $F \in$ $C^{2}(I)$ to the problem (39). The inverse operator $H \mapsto F$ from $C^{2}(I)$ to $C^{2}(I)$ is continuous.

\footnotetext{
${ }^{1}$ This result was obtained by Dr.Galina A.Birulina from Moscow as an answer to the author's question. It would be interesting to get to know, whether this property is characteristic for a $\mathcal{P}$-configuration.
} 


\subsubsection{On a quasi-Cauchy type functional equation.}

Here we consider some functional equation which is formally reminds the equations studied in 3.1.1. The question is the solvability of the equation

$$
F(t)=F\left(a_{1} t\right) F\left(a_{2} t\right) H(t), \quad t \in I^{\prime},
$$

where

$$
0<a_{1}<a_{2}<1,
$$

and $H$ is a given positive continuous function. The case $H(t) \equiv 1$ and $a_{1}^{2}+a_{2}^{2}=1$ has been considered in [KCG, Th. 6.1.2] and the solution $F(t)=e^{\lambda t^{2}}$ has been obtained.

First of all, repeating word for word the proof of Lemma 12, we establish the positiveness of any solution $F$ to equation (41). This makes it possible to reduce equation (41) in an equivalent manner to the linear equation.

$$
(\mathcal{L} G)(t):=G(t)-G\left(a_{1} t\right)-G\left(a_{2} t\right)=\mathcal{H}(t), \quad t \in I^{\prime},
$$

where $G=\ln F, \mathcal{H}=\ln H$. In what follows we denote by $\kappa$ a unique root of the equation

$$
a_{1}^{x}+a_{2}^{x}=1
$$

Denote by $[\kappa]$ and $\{\kappa\}$ the integral and the fractional parts of the number $\kappa$, so that $0<\{\kappa\}<1$. In addition to $\widetilde{C}_{\langle\kappa\rangle}\left(I^{\prime}\right)$, we introduce the linear space

$$
\widetilde{C}_{\langle\kappa, \rho\rangle}\left(I^{\prime}\right)=\left\{u \in C\left(I^{\prime}\right) \mid u(t)=P_{k}(t)+\alpha t^{\kappa}+t^{\kappa+\rho} \gamma(t)\right\},
$$

where $k=[\kappa], 0<\rho<1-\{\kappa\}$, and $\gamma(t)$ is an arbitrary function from $C\left(I^{\prime}\right)$. For the sake of brevity, we agree to use a notation $\alpha=(\partial / \partial t)^{\kappa} u(0) / k ! \kappa$.

Theorem $161^{\circ}$ If $H(t)=1$, then the function

$$
F(t)=e^{\lambda t^{\kappa}}, \quad t \in I^{\prime},
$$

is a unique solution of equation (41) in the space $\widetilde{C}_{\langle\kappa\rangle}\left(I^{\prime}\right)$, satisfying the condition $F(1)=e^{\lambda}$.

$2^{\circ}$ For an arbitrary function $H \in \widetilde{C}_{\langle\kappa, \varepsilon\rangle}\left(I^{\prime}\right), 0<\varepsilon<1-\{\kappa\}$, satisfying the necessary condition

$$
(\partial / \partial t)^{\kappa} H(0)=0,
$$

and for any real number $\lambda$, there is a unique solution $F \in \widetilde{C}_{\langle\kappa, \varepsilon\rangle}\left(I^{\prime}\right)$ of the problem treated in $1^{\circ}$.

Proof. $1^{\circ}$ Let $G \in \widetilde{C}_{\langle\kappa\rangle}\left(I^{\prime}\right)$ be an arbitrary solution of equation (42) with $\mathcal{H}=0$. Then, by definition,

$$
G(t)=P_{k}(t)+t^{\kappa} \gamma(t), \quad t \in I^{\prime},
$$

where $k=[\kappa]$. Assume first that $\kappa \neq k$. It is clear that if $P_{k}(t)=\sum_{j=0}^{k} c_{j} t^{j}$, then $\left(\mathcal{L} P_{k}\right)(t)=\sum_{j=0}^{k} c_{j}\left(1-a_{1}^{j}-a_{2}^{j}\right) t^{j}$ and $\mathcal{L}\left(c t^{\kappa}\right)=c\left(1-a_{1}^{\kappa}-a_{2}^{\kappa}\right) t^{\kappa}=0$ for all constants $c$, by definition of $\kappa$. It follows that the function

$$
\lambda(t)=\gamma(t)-\gamma(0)
$$

satisfies the equation

$$
\lambda(t)-a_{1}^{\kappa} \lambda\left(a_{1} t\right)-a_{2}^{\kappa} \lambda\left(a_{2} t\right)=0 .
$$

Taking into account that $a_{1}^{\kappa}+a_{2}^{\kappa}=1$ and using the same approach as in Subsec.3.2 we conclude that the maximal and the minimal values of the solution $\lambda$ coincide 
(and equal to $\lambda(0)$ ), whence $\lambda(t)=$ const. and, more exactly, $\lambda(t)=0$. This proves the assertion $1^{\circ}$ for $\lambda \neq k$. If $\kappa=k$, then $\gamma(0)=0$ in relation (43), and we arrive at the same result by repeating the same arguments word for word. This completes the proof of $1^{\circ}$.

$2^{\circ} \quad$ First of all, let us show that if a function $G \in \widetilde{C}_{\langle\kappa\rangle}$ solves the equation $\mathcal{L} G=H$ with $H \in \widetilde{C}_{\langle\kappa\rangle}$, then $(\partial / \partial t)^{\kappa} H(0)=0$. Indeed, let

$$
\begin{aligned}
& G=\sum_{j=0}^{k} c_{j} t^{j}+c t^{\kappa}+t^{\kappa} \mu(t) \quad \text { and } \\
& H=\sum_{j=0}^{k} b_{j} t^{j}+b t^{\kappa}+t^{\kappa} \nu(t)
\end{aligned}
$$

where $b$ and $c$ are constants and $\mu(0)=\nu(0)=0$. It is obvious that

$$
b=c\left(1-a_{1}^{\kappa}-a_{2}^{\kappa}\right)=0,
$$

if $\kappa \neq k$, and $b_{k}=c_{k}\left(1-a_{1}^{k}-a_{2}^{k}\right)=0$, if $\kappa=k$. To prove the assertion it remains to present a solution $G$ of the equation $\mathcal{L} G=H$, for any given $H \in \widetilde{C}_{\langle\kappa, \varepsilon\rangle}\left(I^{\prime}\right)$. Write down the functions $G$ and $H$ in the form

$$
\begin{aligned}
& G=\sum_{1}^{k} c_{j} t^{j}+\alpha t^{\kappa}+\beta t^{\kappa+\varepsilon}+t^{\kappa+\varepsilon} \mu(t), \quad \mu(0)=0 \\
& H=\sum_{1}^{k} b_{j} t^{j}+\beta_{1} t^{\kappa+\varepsilon}+t^{\kappa+\varepsilon} \nu(t), \quad \nu(0)=0 .
\end{aligned}
$$

If we choose $c_{j}=b_{j} /\left(1-a_{1}^{j}-b_{1}^{j}\right), \beta=\beta_{1} /\left(1-a_{1}^{\kappa+\varepsilon}-a_{2}^{\kappa+\varepsilon}\right)$, then what remains to do is to choose, as $\mu(t)$, a solution of the problem

$$
\mathcal{L}\left(t^{\kappa+\varepsilon} \mu\right)=t^{\kappa+\varepsilon} \nu, \quad \mu(0)=0 .
$$

As $\mathcal{L}\left(t^{\kappa+\varepsilon} \mu\right)=t^{\kappa+\varepsilon}\left[\mu(t)-a_{1}^{\kappa+\varepsilon} \mu\left(a_{1} t\right)-a_{2}^{\kappa+\varepsilon} \mu\left(a_{2} t\right)\right]$, problem (44) takes the form

$$
\mu(t)-a_{1}^{\kappa+\varepsilon} \mu\left(a_{1} t\right)-a_{2}^{\kappa+\varepsilon} \mu\left(a_{2} t\right)=\nu(t), \quad \mu(0)=0 .
$$

But, by definition of $\kappa$, the relation

$$
a_{1}^{\kappa+\varepsilon}+a_{2}^{\kappa+\varepsilon}<1
$$

holds, and this means that the norm of the linear operator in the space $C\left(I^{\prime}\right)$

$$
\mathcal{L}_{\varepsilon}: \mu(t) \mapsto a_{1}^{\kappa+\varepsilon} \mu\left(a_{1} t\right)+a_{2}^{\kappa+\varepsilon} \mu\left(a_{2} t\right)
$$

is less then one. It follows that the sum of the Neumann series $\sum_{n=0}^{\infty} \mathcal{L}_{\varepsilon}^{n} \nu$ with $\mathcal{L}_{\varepsilon}^{0}=i d$ solves the equation in (45). The boundary condition $\mu(0)=0$ is valid, as $\nu(0)=0$ and so $\left(\mathcal{L}_{\varepsilon}^{n} \nu\right)(0)=0$ for all $n=1,2, \ldots$. This completes the proof of Theorem 16.

\subsection{On some integral equations relating to a geometric problem}

In this subsection we deal with one of the typical problems in integral geometry: to reconstruct a function in a given domain $D$ from the values of its integrals over a family $\left\{D_{q}\right\}$ of subdomains in $D$. Such problems are always of interest first of 
all by themselves as objects of pure analysis, but also in connection with possible applications in practical disciplines. The most remarkable example of such a connection is the famous Radon problem and tomography. A peculiarity of the geometric problems considering in this paper is that in contrast with a general tradition, we deal with boundary domains $D$ and the statement of these problems as well as the corresponding results are closely connected with both local and global properties of the boundaries $\partial D$. This connection is realized by means of a semigroup $\Phi_{D}$ of maps from $\partial D$ into itself we associate with the problem in question. On the other hand, as will be seen, these problems can be reduced to Cauchy type functional equations on the intervals $I$ and $I^{\prime}$, distinguishing from each other by configurations of maps generating these equations. These maps also generate a semigroups $\Phi_{I}, \Phi_{I^{\prime}}$. We introduce a special isomorphism between the boundary $\partial D$ and (each of) the intervals $I$ or $I^{\prime}$, and we show that it can be extended up to isomorphism of the above semigroups $\Phi_{D}$ and $\Phi_{I}$. This makes it possible to formulate (and to obtain) all the geometric results in terms of the semigroup $\Phi_{D}$, by knowing the conditions of solvability of Cauchy type functional equations in terms of semigroups $\Phi_{I}$ and $\Phi_{I^{\prime}}$. We consider separately two situations.

\subsubsection{The case of a $Z$ - configuration.}

Let $\bar{l}_{1}$ and $\bar{l}_{2}$ be smooth nonsingular transversal vector fields in a disk $B \subset \mathbb{R}^{2}$. Consider a curvilinear parallelogram $D=O A_{1} O^{\prime} A_{2}$ in $B$ whose sides $O A_{1}, O^{\prime} A_{2}$ and $O A_{2}, O^{\prime} A_{1}$ are trajectories of the vector fields $\bar{l}_{1}$ and $\bar{l}_{2}$, respectively. Let $\Gamma=O O^{\prime}$ be a quasidiagonal in $D$, i.e. a smooth curve having no common points with the sides of $D$ and being a trajectory of the vector field $\bar{l}=r_{1} \bar{l}_{1}+r_{2} \bar{l}_{2}$ with some positive constant $r_{1}$ and $r_{2}$. It is easily seen that, given vector fields $\bar{l}_{1}, \bar{l}_{2}$ and a point $O$, there are some points $A_{1}, A_{2}$ and $O^{\prime}$ such that all the above conditions are satisfied.

Introduce in $D$ projections

$$
\pi_{1}: D \rightarrow O A_{1}, \quad \pi_{2}: D \rightarrow O A_{2}
$$

along the vector fields $\bar{l}_{2}$ and $\bar{l}_{1}$, respectively. Given an arbitrary point $q \in \Gamma$, denote by $D_{q}$ the curvilinear parallelogram $q q_{1} O q_{2}$ where $q_{1}=\pi_{1} q$ and $q_{2}=\pi_{2} q$. Let us associate with any function $f$ in $D$ the integrals

$$
(\mathcal{A} f)(q)=\int_{D_{q}} f d \sigma, \quad q \in \Gamma,
$$

were $\sigma$ denotes a measure on $B$. The above mentioned geometric problem of reconstructing a function in $\bar{D}$ takes now a completely definite form:

given a function $h$ on $\Gamma$, to find a function $f$ in $D$ such that

$$
\mathcal{A} f(q)=h(q), \quad q \in \Gamma \text {. }
$$

For obtaining reach in content results related to equation (46) we will study it in the framework of the general problem stated in [GGV] for a wide class of integral equations: for what spaces of functions $f$ and $h$ is the map $\mathcal{A}: f \mapsto h$ one-to-one, and what functions $h(q)$ may be represented by the integral in $(\mathcal{A} f)(q)$. Restricting ourselves to continuous functions on $D$ and on $\Gamma$ we find immediately that the kernel of the operator $\mathcal{A}$ is infinite-dimensional. On the other hand, as will be seen, for an arbitrary function $f \in C(\bar{D})$, its image $(\mathcal{A} f)(q)$ belongs to the space $\mathcal{H}(\Gamma)$ of all twice continuously differentiable on $\Gamma$ functions vanishing with the first derivative at 
the point 0 . Therefore, the best possible solution of the problem in question consists in describing subspaces $\mathcal{F}(D) \subset C(\bar{D})$ for which the map

$$
\mathcal{A}: \mathcal{F}(D) \rightarrow \mathcal{H}(\Gamma)
$$

is one-to-one. Among various candidates for the role of $\mathcal{F}(D)$ we have chosen a wide class of subspaces in $C(\bar{D})$ arising naturally when solving a wide class of boundary problems for higher order hyperbolic equations (see Subsec. 4.3).

Definition 5. Given a smooth vector field $\bar{l}$ in $B$, we denote by $C_{\langle\overline{\langle}\rangle}(D)$ the subset of functions in $C(\bar{D})$, remaining constant along each trajectory of the field $\bar{l}$.

As is well known the space $C_{\langle\bar{l}\rangle}(D)$ admits a very simple description. Let $y=$ $\left(y_{1}, y_{2}\right)$ be a coordinate system in $B$ and

$$
\bar{l}=\left\{\omega_{1}(y), \omega_{2}(y)\right\}, \quad y \in B
$$

be a coordinate form of $\bar{l}$. Denote by $\zeta(y)$ a smooth function without critical points solving the first order differential equation

$$
\omega_{1}(y)\left(\partial / \partial y_{1}\right) \zeta+\omega_{2}(y)\left(\partial / \partial y_{2}\right) \zeta=0 .
$$

Then the space $C_{\langle\bar{l}\rangle}(D)$ consists of all functions $f(y)=F(\zeta(y))$ with $F$ being arbitrary continuous function on the range of $\zeta$.

Now everything is ready to formulate our first geometric result.

Theorem 17 Let $\bar{l}_{1}, \bar{l}_{2}$ and $\bar{l}$ be a triple of smooth nonsingular mutually transversal vector fields in a dick $B \in \mathbb{R}^{2}$ and let $D$ be an above parallelogram constructed by $\bar{l}_{1}$ and $\bar{l}_{2}$. Assume that the corresponding quasidiagonal $\Gamma$ is a twice differentiable nonsingular curve which is transversal to $\bar{l}$ everywhere and to $\bar{l}_{1}$ and $\bar{l}_{2}$ of the point $O$. Then for an arbitrary function $H \in \mathcal{H}(\Gamma)$ there is a unique solution $f \in C_{\bar{\zeta}\rangle}(D)$ of equation (46). The inverse operator $\mathcal{A}^{-1}$ is a continuous operator from $\mathcal{H}(\Gamma)$ to $C_{\langle\bar{l}\rangle}(D)$.

Proof. For the sake of brevity we consider the situation with constant vector fields $\bar{l}_{1}, \bar{l}_{2}$ and $\bar{l}$. Without loss of generality we assume that the vector fields $\bar{l}_{1}, \bar{l}_{2}$ are parallel to the coordinate axes $x_{1}$ and $x_{2}$, respectively, and consider as $D$ a square whose two sides coincide with the intervals $\left\{x_{1} \mid 0 \leq x_{1} \leq 1\right\}$ and $\left\{x_{2} \mid 0 \leq x_{2} \leq 1\right\}$ on the axes $x_{1}$ and $x_{2}$, respectively. Let

$$
x_{1}=\delta_{1}(t), \quad x_{2}=\delta_{2}(t), \quad t \in I^{\prime},
$$

be an arbitrary parametric representation of $\Gamma$, so that

$$
\delta_{1}(0)=\delta_{2}(0)=0
$$

If $\bar{l}=\left(\omega_{1}, \omega_{2}\right)$ with $\omega_{1}>0, \omega_{2}<0$, then equation (46) in the coordinate form looks as follows:

$$
\int_{0}^{\delta_{1}(t)} \int_{0}^{\delta_{2}(t)} f\left(\omega_{1} x_{2}-\omega_{2} x_{1}\right) d x_{2} d x_{1}=H(t), \quad t \in I .
$$

To see this it suffices to note that the function $\zeta\left(x_{1}, x_{2}\right)=\omega_{1} x_{2}-\omega_{2} x_{1}$ solves equation (47).

Introduce the new unknown function $F(t)$ as a solution to the problem

$$
F^{\prime \prime}(t)=f(t), \quad F(0)=F^{\prime}(0)=0,
$$


and substitute $F^{\prime \prime}$ for $f$ in (49). After repeated integration by parts we arrive at the following functional equation for the function $F$ :

$$
F\left(\omega_{1} \delta_{2}(t)-\omega_{2} \delta_{1}(t)\right)-F\left(\omega_{1} \delta_{2}(t)\right)-F\left(-\omega_{2} \delta_{1}(t)\right)=H(t) .
$$

Since the map $f \mapsto F$ is one-to-one, to prove the theorem, it remains to establish the unique solvability of equation (51). Note that, by assumptions of the theorem, we have $\left(\delta_{1}^{\prime} \delta_{2}^{\prime}\right)(0)>0$, which means that

$$
\omega_{1} \delta_{2}^{\prime}(0)-\omega_{2} \delta_{1}^{\prime}(0)>0 .
$$

The transversality condition $\Gamma \pitchfork \bar{l}$ guarantees the inequality $\omega_{1} \delta_{2}^{\prime}(t)-\omega_{2} \delta_{1}^{\prime}(t)>0$ at any point $t \in I^{\prime}$. On the other hand, differentiating relation (49) leads to the differentiability of the function $H$ and to the relation

$$
\delta_{1}^{\prime}(t) \int_{0}^{\delta_{2}(t)} f\left(\omega_{1} x_{2}-\omega_{1} \delta_{1}(t)\right) d x_{2}+\delta_{2}^{\prime}(t) \int_{0}^{\delta_{1}(t)} f\left(\omega_{1} \delta_{2}(t)-\omega_{2} x_{1}\right) d x_{1}=H^{\prime}(t) .
$$

It follows, by (48), that $H^{\prime}(0)=0$. To see that the function $H$ is twice differentiated, it suffices to represent the left hand-side of the late relation in the form

$$
\left(-\delta_{1}^{\prime}(t) / \omega_{2}\right) \int_{-\omega_{2} \delta_{1}(t)}^{\omega_{1} \delta_{2}(t)-\omega_{2} \delta_{1}(t)} f(z) d z+\left(\delta_{2}^{\prime}(t) / \omega_{1}\right) \int_{\omega_{1} \delta_{2}(t)}^{\omega_{1} \delta_{2}(t)-\omega_{2} \delta_{1}(t)} f(z)
$$

Thus, equation (51) is nothing, but a Cauchy type functional equation on $I^{\prime}$, corresponding to a $Z$-configuration and satisfying all conditions of Theorem 2. By this theorem, for an arbitrary function $H \in C^{2}\left(I^{\prime}\right)$, satisfying the hypothesis $H^{\prime}(0)=0$, there is a unique solution $F \in C_{\langle 1+r\rangle}\left(I^{\prime}\right)$ of equation (51), and, by Theorem 4, this function $F$ belongs to the space $C^{2}\left(I^{\prime}\right)$. This proves the existence and uniqueness of a function $f \in C_{\langle\bar{l}\rangle}(D)$, solving equation (49), and completes the proof of Theorem 17 in the part of the existing of the inverse operator $\mathcal{A}^{-1}: C^{2}(\Gamma) \rightarrow C(\bar{D})$. As for the continuity of $\mathcal{A}^{-1}$, it follows immediately from the Banach closed graph theorem which, in turn, is a consequence of the elementary inequality $\left\|A f, C^{2}\right\|<$ const $\|f, C\|$, proved above.

Remark The reader easily write down the solution of equation (49) in an explicit form, using the result of Theorem 1.

\subsubsection{The case of a $\mathcal{P}$ - configuration.}

In this Subsection a geometric problem analogous to that considered above is studied. But this time a dynamical system associated with this problem turns out to be much more complicated, and the result obtained is closely connected with a new boundary problem for higher order hyperbolic differential equations in a bounded domain in $\mathbb{R}^{2}$, see Subsec. 3.3.

As above, let $\bar{l}_{1}$ and $\bar{l}_{2}$ be some nonsingular smooth transversal vector fields in a disk $B \subset \mathbb{R}^{2}$. Consider a curvilinear triangle $D=O A_{1} A_{2}$, whose sides $O A_{1}$ and $O A_{2}$ coincide with trajectories of the vector fields $\bar{l}_{1}$ and $\bar{l}_{2}$, respectively, and the side $\Gamma=A_{1} A_{2}$ is an arbitrary nonsingular smooth curve transversal to both fields $\bar{l}_{1}, \bar{l}_{2}$ at the points $A_{1}$ and $A_{2}$. In addition, the closure $\bar{D}$ of the domain $D$ is supposed to satisfy the following topological hypotheses:

(i) For any point $p \in \bar{D}$, a trajectory of $\bar{l}_{j}$ passing through $p$ meets $O A_{k}$ at a point $\pi_{k} p, j \neq k, 1 \leq j, k \leq 2$. 
(ii) The set $\bar{D}$ is $\bar{l}_{j}$ - convex, $j=1,2$. This means that if given points $p$ and $q$ in $\bar{D}$ lie on some trajectory $\gamma_{j}$ of the field $\bar{l}_{j}$, then all the points $r \in \gamma_{j}, j=1,2$ between $p$ and $q$ belong to $\bar{D}$.

Given an arbitrary point $q \in \Gamma$, let $D_{q}$ be a curvilinear parallelogram $q q_{1} O q_{2}$.

Hypotheses (i) and (ii) guarantee the inclusion $D_{q} \subset \bar{D}$ for all $q \in \Gamma$ and something more. Namely, let $x=\left(x_{1}, x_{2}\right)$ be a coordinate system in $B$ with the origin at the point $O$. Let

$$
x_{1}=\alpha_{1}(z), \quad x_{2}=\alpha_{2}(z), \quad z \in I,
$$

be an arbitrary parametric representation of $\Gamma$ and let $\alpha(z)=\left(\alpha_{1}(z), \alpha_{2}(z)\right)$. Then the functions $\alpha_{1}(z)$ and $\alpha_{2}(z)$ satisfy the conditions

$$
\alpha_{1}^{\prime}(z) \geq 0, \quad \alpha_{2}^{\prime}(z) \leq 0, \quad \text { and } \quad \alpha_{1}^{\prime}(z)-\alpha_{2}^{\prime}(z)>0
$$

This fact is not trivial (see [P4] with respect to the proof).

The geometric problem under consideration takes this time the form of the same integral equation

$$
(\mathcal{A} f)(q):=\int_{D_{q}} f d \sigma=h(q), \quad q \in \Gamma,
$$

as in Subsec. 4.2.1, but with a different domain $D_{q}$. All the discussions around the statement of problem (46) are relevant to problem (53). In particular, the righthand side $h(q)$ in (53) should be a twice differentiable function on $\Gamma$ and satisfy the periodicity condition

$$
h\left(A_{1}\right)=h\left(A_{2}\right)=0 .
$$

The latter follows immediately from (53). We denote the space of all such functions by $\mathcal{H}_{0}(\Gamma)$. To formulate the result, related to the solvability of equation $(53)$ in an invariant geometric form (not passing to any coordinate form of (53)) it is necessary to generalize the above dynamical approach by considering certain semigroups of maps from some curves into themselves. It is interesting to trace the transition from a pure geometric problem to a Cauchy type functional equation (what includes also a translation from a geometric language to an analytic one).

Introduce the vector field $\bar{l}=r_{1} \bar{l}_{1}+r_{2} \bar{l}_{2}$, where $r_{1}$ and $r_{2}$ are positive constants. In addition to the projections $\pi_{j}, j=1,2$, along the vector fields $\bar{l}_{k}, j \neq k$, we consider the projection $\pi_{\bar{l}}: \bar{D} \rightarrow \Gamma$ along the field $\bar{l}$. In other words, given an arbitrary point $p \in \bar{D}$, its projection $\pi_{\bar{l}} p$ coincides with the intersection of the curve $\Gamma$ and the trajectory of the field $\bar{l}$, passing through $p$. Introduce two maps from $\Gamma$ into itself,

$$
\zeta_{1}=\pi_{\bar{l}} \circ \pi_{1} \quad \text { and } \quad \zeta_{2}=\pi_{\bar{l}} \circ \pi_{2}
$$

and denote by $\Phi_{\zeta}$ the semigroup of maps generated by $\zeta_{1}$ and $\zeta_{2}$, which is analogous to the semigroup $\Phi_{\delta}$ considered in Sec. 2. As in Subsec. 2.2, we define by means of the maps $\zeta_{1}$ and $\zeta_{2}$ an orbit in $\Gamma$ as a sequence of points $\mathcal{O}=\left(q_{1}, q_{2}, \ldots, q_{n}, \ldots\right)$ such that

$$
q_{k+1}=\zeta_{j_{k}}\left(q_{k}\right), k=1,2, \ldots,
$$

and the set of all such orbits we denote by $\mathcal{O}_{\zeta}$. We introduce the guiding sets

$$
\mathcal{T}_{j}=\left\{q \in \Gamma \mid \bar{l}_{j}(q) \in T_{q}(\Gamma)\right\}, \quad j=1,2, \quad \text { and } \quad \mathcal{T}=\mathcal{T}_{1} \cup \mathcal{T}_{2},
$$

where $T_{q}(\Gamma)$ stands for the tangent space to $\Gamma$ at the point $q$. Repeating literally what was said in Subsec. 2.2 we define periodic, $\mathcal{T}$ - guided, and $\mathcal{T}$ - proper orbits corresponding to the semigroup $\Phi_{\zeta}$. Finally, we denote by $\mathfrak{N}_{\zeta}^{\mathcal{T}}$ the set of $\mathcal{T}$-proper $\mathcal{T}$ - guided periodic orbits of the type in question. By analogy with condition (13), 
we assume that, if both sets $\mathcal{T}_{1}$ and $\mathcal{T}_{2}$ are infinite, then arbitrary points $\tau_{1} \in \mathcal{T}_{1}^{\prime}$ and $\tau_{2} \in \mathcal{T}_{2}^{\prime}$ are situated on the curve $\Gamma$ in the order $A_{1}, \tau_{1}, \tau_{2}, A_{2}$. The main result, related to the problem in consideration, is formulated as the following assertion.

Theorem 18 If all the above hypotheses related to the domain $D$, the curve $\Gamma$ and vector fields $\bar{l}, \bar{l}_{1}$ and $\bar{l}_{2}$ are fulfilled, then, for an arbitrary function $h \in \mathcal{H}_{0}(\Gamma)$, there is a unique solution $f \in C_{\langle\bar{\zeta}\rangle}(D)$ of equation (53) if and only if $\mathfrak{N}_{\zeta}^{\mathcal{T}}=\emptyset$. The inverse operator $A^{-1}: h \mapsto f$ is continuous from $\mathcal{H}_{0}(\Gamma)$ to $C_{\langle\bar{l}\rangle}(D)$.

Proof. Without loss of generality, we assume that the vector fields $\bar{l}_{1}$ and $\bar{l}_{2}$ are parallel to the coordinate axes $x_{1}$ and $x_{2}$, respectively, and consider as $D$ a curvilinear triangle whose two sides coincide with the intervals $\left\{x_{1} \mid 0 \leq x_{1} \leq 1\right\}$ and $\left\{x_{2} \mid 0 \leq x_{2} \leq 1\right\}$ on the axes $x_{1}$ and $x_{2}$, respectively. Under this assumption, the vector field $\bar{l}$ takes the form $\bar{l}=r_{1} \bar{l}_{1}+r_{2} \bar{l}_{2}$. With respect to the third side $\Gamma$ of the triangle $D$, it is assumed to be a smooth nonsingular curve transversal to the coordinate axes. Let

$$
x_{1}=\alpha_{1}(z), \quad x_{2}=\alpha_{2}(z), \quad z \in I
$$

be an arbitrary parametric representation of the curve $\Gamma$. Introduce the notation

$$
\alpha(z)=\left(\alpha_{1}(z), \alpha_{2}(z)\right),
$$

and assume that $\alpha(0)=(0,1)$. Then, as was explained above, relations (52) are valid. It is convenient to treat $\alpha$ as a map: $I \rightarrow \Gamma$ which is invertible, by (52). As was explained above, the space $C_{\langle\bar{l}\rangle}(\bar{D})$ consists of the functions $f\left(r_{2} x_{1}-r_{1} x_{2}\right)$ with $f$ being an arbitrary continuous function on the closed interval $I_{T}=\left(-r_{1}, r_{2}\right)$. Introduce the functions

$$
\omega(x)=r_{2} x_{1}-r_{1} x_{2}, \quad \omega_{1}(x)=\omega\left(x_{1}, 0\right), \omega_{2}(x)=\omega\left(0, x_{2}\right)
$$

in $\bar{D}$, and denote by $\omega_{\Gamma}$ the restriction of $\omega$ to $\Gamma$. (I do not use consciously the explicit values of the functions $\omega_{1}$ and $\omega_{2}$ to give to the reader a possibility to observe the main springs of the proof not connected with the constancy of the vector field $\bar{l}$ ). It is obvious, that the function

$$
\sigma(z)=\omega_{\Gamma} \circ \alpha(z): I \rightarrow I_{T}
$$

is invertible and surjective. Indeed, the relation

$$
\sigma^{\prime}(z)=\omega_{x_{1}}(\alpha) \alpha_{1}^{\prime}(z)+\omega_{x_{2}}(\alpha) \alpha_{2}^{\prime}(z)>0, \quad z \in I,
$$

holds in view of the inequalities

$$
\omega_{x_{1}}>0, \quad \omega_{x_{2}}<0, \quad \alpha_{1}^{\prime}(z) \geq 0, \quad \alpha_{2}^{\prime}(z) \leq 0,
$$

and this proves the invertibility. As to the surjectivity, take a point $q \in \bar{D}$ such that $\omega(q)=-r_{2}$. The trajectory of the vector field $\bar{l}$ passing through $q$ meets $\Gamma$ at a point $q_{-}$. Therefore $\omega_{\Gamma}\left(q_{-}\right)=-r_{2}$. In just the same way we determine a point $q_{+} \in \Gamma$, for which $\omega_{\Gamma}\left(q_{+}\right)=-r_{1}$. This completes the proof of the assertion.

Now, in the coordinate form, equation (53) looks as follows:

$$
\int_{0}^{\alpha_{1}(z)} \int_{0}^{\alpha_{2}(z)} f(\omega(x)) d x_{2} d x_{1}=h(z), \quad z \in I .
$$


Applying the one-to-one change (50) of an unknown function $f \rightarrow F$ we arrive at the following equivalent functional equation for the function $F$ :

$$
F\left(\omega_{\Gamma} \circ \alpha(z)\right)-F\left(\omega_{1} \circ \alpha(z)\right)-F\left(\omega_{2} \circ \alpha(z)\right)=H(z),
$$

Introduce the new variable $t=\sigma(z), t \in I_{T}$. Remembering that, by the definition of the map $\zeta$,

$$
\omega_{j} \circ \alpha=\omega_{\Gamma} \circ \zeta_{j} \circ \alpha, j=1,2 .
$$

we can rewrite equation (54) in the form

$$
F(t)-F\left(\omega_{\Gamma} \circ \zeta_{1} \circ \omega_{\Gamma}^{-1}(t)\right)-F\left(\omega_{\Gamma} \circ \zeta_{2} \circ \omega_{\Gamma}^{-1}(t)\right)=h\left(\sigma^{-1}(t)\right), \quad t \in I_{T} .
$$

Let

$$
\delta_{j}(t)=\omega_{\Gamma} \circ \zeta_{j} \circ \omega_{\Gamma}^{-1}(t), \quad t \in I_{T} .
$$

Then, the last functional equation takes the form

$$
F(t)-F\left(\delta_{1}(t)\right)-F\left(\delta_{2}(t)\right)=(h \circ \sigma)(t), \quad t \in I_{T} .
$$

We are now going to show that this equation is a Cauchy type functional equation. This is an immediate corollary of the following lemma.

Lemma $191^{\circ} \quad$ Both maps $\delta_{1}$ and $\delta_{2}$ are nondecreasing functions;

$2^{\circ}$ the range of $\delta_{1}\left(\right.$ resp. $\left.\delta_{2}\right)$ is the closed interval $\left(0, r_{1}\right)\left(\right.$ resp. $\left.\left(-r_{2}, 0\right)\right)$;

$3^{\circ}$ the relation

$$
\delta_{1}(t)+\delta_{2}(t)=t
$$

holds for all $t \in I_{T}$.

Proof. $1^{\circ}$ To simplify notation consider the case of $\delta_{1}$ only. Note that, by the definition, $\omega_{\Gamma}^{-1}(t)=\alpha(z)$, so that $\delta_{1}(t)=\omega\left(\zeta_{1}(\alpha(z))\right)$, where $t=\sigma(z)$. By the definition of the map $\zeta_{1}$ it follows that $\delta_{1}(t)=\omega\left(\alpha_{1}(z), 0\right)$. Thus, $\delta_{1}^{\prime}(t)=\omega_{x_{1}}\left(\alpha_{1}, 0\right) \alpha_{1}^{\prime}(z) \geq 0$, by (52).

$2^{\circ} \quad$ By the definition of the map $\zeta_{1}: \Gamma \rightarrow \Gamma$ we have

$$
\delta_{1}(t)=\omega\left(\pi_{1} \circ \alpha(z), 0\right), \quad \text { where } t=\sigma(z) .
$$

As all the functions $\delta_{1}, \omega\left(x_{1}, 0\right)$ and $\alpha_{1}(z)$ are nondecreasing, the range of the map $\delta_{1}$ coincides with the interval $\left(\omega\left(\alpha_{1}(0), 0\right), \omega\left(\alpha_{1}(1), 0\right)\right)$, i.e. $\mathcal{R}\left[\delta_{1}\right]=\left(-r_{1}, 0\right)$. In just the same way one can prove that $\mathcal{R}\left[\delta_{2}\right]=\left(0, r_{2}\right)$.

$3^{\circ}$ To prove the assertion we use again relation (57). By linearity of the function $\omega$, it follows that

$$
\delta_{1}(t)+\delta_{2}(t)=\omega\left(\alpha_{1}(z), 0\right)+\omega\left(0, \alpha_{2}(z)\right)=\omega\left(\alpha_{1}(z), \alpha_{2}(z)\right)=(\omega \circ \alpha)\left(\sigma^{-1}(t)\right)=t .
$$

This lemma makes it possible to use the result of Theorem 5, when studying the solvability properties of equation (56). By this theorem we have to consider the corresponding semigroup $\Phi_{\delta}$ with $\delta=\left(\delta_{1}, \delta_{2}\right)$, to introduce the guiding sets

$$
\mathcal{T}_{j}^{\delta}=\left\{t \in I_{T} \mid \delta_{j}^{\prime}(t)=0\right\}, \quad j=1,2,
$$

and the corresponding sets of $\mathcal{T}^{\delta}$ - guided and $\mathcal{T}^{\delta}$ - proper orbits in $I_{T}$. Then, as is known from Theorem 5 , the condition $\mathfrak{N}_{\delta}^{\mathcal{T}_{\delta}}=\emptyset$ is necessary and sufficient for equation (56) to be uniquely solvable.

Returning to the proof of Theorem 18, we conclude that to complete this proof it suffices to show that the relations

$$
\mathfrak{N}_{\delta}^{\mathcal{I}_{\delta}}=\emptyset \quad \text { and } \quad \mathfrak{N}_{\zeta}^{\mathcal{T}_{\zeta}}=\emptyset
$$

hold only simultaneously.

This follows immediately from the following lemma. 
Lemma 20 The $\omega$-image of any $\left(\mathcal{T}_{\zeta^{-}}\right.$guided, $\mathcal{T}_{\zeta^{-}}$proper $)$orbit in $\mathcal{O}_{\zeta}$ is a $\left(\mathcal{T}_{\delta^{-}}\right.$ guided, $\mathcal{T}_{\delta}$ - proper) orbit in $\mathcal{O}_{\delta}$, and, conversely, any $\left(\mathcal{T}_{\delta}\right.$ - guided, $\mathcal{T}_{\delta}$ - proper) orbit in $\mathcal{O}_{\delta}$ is an $\omega$ - image of a $\left(\mathcal{T}_{\zeta}\right.$-guided, $\mathcal{T}_{\delta}$ - proper $)$ orbit in $\mathcal{O}_{\zeta}$.

Proof. It is sufficient to consider only two-element orbits. Let $(q, \widehat{q})$ be an orbit from $\mathcal{O}_{\zeta}$, that is $\widehat{q}=\zeta_{j}(q)$ for some $j=1,2$. Then, by (55),

$$
\omega(\widehat{q})=\omega \circ \zeta_{j}(q)=\left(\delta_{j} \circ \omega\right)(q),
$$

which means that the ordered pair $(\omega(q), \omega(\widehat{q}))$ is an orbit from $\mathcal{O}_{\delta}$. If $(t, \widehat{t})$ is an orbit from $\mathcal{O}_{\delta}$, i.e. $\widehat{t}=\delta_{j}(t)$ for some $j=1,2$, then $\omega_{\Gamma}^{-1}(\widehat{t})=\zeta_{j} \circ \omega_{\Gamma}^{-1}(t)$, by $(55)$, which means that $(t, \widehat{t})$ is the $\omega$ - image of an orbit $\left(\omega_{\Gamma}^{-1}(t), \omega_{\Gamma}^{-1}(\widehat{t})\right)$ from $\mathcal{O}_{\zeta}$. This proves the lemma in the case of nonspecific orbits.

To prove the lemma it remains to show that the $\omega$-images of the guided sets $\left(\mathcal{T}_{\zeta}\right)_{j}$ coincide with the guided sets $\left(\mathcal{T}_{\delta}\right)_{j}, j=1,2$. To this end, take an arbitrary point $q$ from the guiding set $\left(\mathcal{T}_{\zeta}\right)_{1}$, for definiteness. This means that $q=\alpha(z)$ for a (single) $z \in I$ and $\alpha_{1}^{\prime}(z)=0$. Let $t=\omega(q)$. Then $t=\sigma(z)=(\omega \circ \alpha)(z)$ and, by (55),

$$
\delta_{1}^{\prime}(\sigma(z)) \sigma^{\prime}(z)=\omega_{x_{1}}\left(\alpha_{1}(z), 0\right) \cdot \alpha_{1}^{\prime}(z) .
$$

As $\sigma^{\prime}>0$ and $\omega_{x_{1}}>0$, the relations

$$
\alpha_{1}^{\prime}(z)=0, \quad \text { and } \quad \delta_{1}^{\prime}(t)=0 \quad \text { for } t=\sigma(z)
$$

are equivalent. This completes the proof of the lemma and, moreover, the proof of Theorem 18 in the part of the solvability of equation (53). As for the boundedness of the inverse operator $\mathcal{A}^{-1}$, it suffices to refer to the proof of the analogous assertion in Theorem 17. The necessity of the condition $\mathfrak{N}_{\zeta}^{\mathcal{T}}=\emptyset$ is actually proved when proving the correspondent part of Theorem 6 .

\subsection{On the solvability of boundary problem for hyperbolic differ- ential equations.}

As another application of the results relating to a Cauchy type functional equation we consider now an arbitrary homogeneous strictly hyperbolic differential operator $P\left(\partial_{x}, \partial_{y}\right)$ of the third order in the $(x, y)$ - plane $\mathbb{R}^{2}$. By definition it is an arbitrary differential operator of the form

$$
P=\left(\partial / \partial \bar{l}_{1}\right)\left(\partial / \partial \bar{l}_{2}\right)\left(\partial / \partial \bar{l}_{3}\right)
$$

where $\bar{l}_{1}, \bar{l}_{2}$ and $\bar{l}_{3}$ are arbitrary mutually transversal vector fields in $\mathbb{R}^{2}$. In the framework of the classical theory of partial differential equations such operators arise only in connection with the Cauchy problem for arbitrary hyperbolic equations. A systematical study of boundary problems for such equations in bounded domains was begin less then 10 years ago (see [P5]), although some particular equations of this type arisen earlier in connection with some problems relating to linearized flow of a chemically relaxing gas $([\mathrm{Ch}])$.

In this Subsection we consider for the sake of brevity only differential operators of the above type with constant coefficients although the boundary problems can be easily formulated in an invariant form (not using coordinate systems) for analogous operators with variable coefficients. As will be seen, all the known up to now problems in question turn out to be equivalent to one of Cauchy type functional equations (responding either $Z$ - or $\mathcal{P}$ - configurations). Thus, the general theory of functional equations, its language and its methods, become an essential technical tool when solving boundary problems for partial differential equations, and hence, to be applied in different practical disciplines. 


\subsubsection{Formulation of boundary problems.}

Let $\bar{l}_{1}, \bar{l}_{2}$ and $\bar{l}_{3}$ be an above triple of smooth nonsingular mutually transversal vector fields in a disk $B \subset \mathbb{R}^{2}$. (The reader not familiar with this differentialgeometric terminology may think on a triple constant nonparallel vector fields). Take any point $O \in B$ and consider six semitrajectories of these vector fields, beginning at $O$. All this rays are nothing but characteristics of the operator $P$. Take an arbitrary triple of neighboring rays $l_{1}, l_{2}$ and $l_{3}$ lying on trajectories of the vector fields $\bar{l}_{1}, \bar{l}_{2}$ and $\bar{l}_{3}$, respectively, and choose arbitrary points $A_{1} \in l_{1}, A_{2} \in l_{2}$, and $A_{3} \in l_{3}$. Assume without loss of generality that the ray $l_{2}$ lies between $l_{1}$ and $l_{3}$ (i.e. $\bar{l}_{2}=\lambda_{1} \bar{l}_{1}+\lambda_{3} \bar{l}_{3}$ with $\lambda_{1}>0, \lambda_{3}>0$, see Fig. 1$)$.

We associate with the differential operator $P$ canonically two different bounded domains $D_{1}$ and $D_{2}$, and they are just domains where different boundary problems for the equation $P u=f$ will be considered.

The domain $D_{1}$ is determined as the curvilinear triangle $A_{1} O A_{3}$ whose third side $\Gamma=A_{1} A_{3}$ is an arbitrary nonsingular $C^{2}$ - curve.

The domain $D_{2}$ is determined as the characteristic parallelogram $A_{1} O A_{2} O^{\prime}$, whose sides $A_{1} O^{\prime}$ and $A_{2} O^{\prime}$ are trajectories of the vector fields $\bar{l}_{1}$ and $\bar{l}_{2}$, respectively.

Remark It is clear that given a point $O$ there are six different domains of the $D_{1}$ - and $D_{2}$-types. In none of domains of different types the equation $P u=f$ in question has been studied. In the following two sections we study separately the boundary problems of both types.

\subsubsection{First partly characteristic problem.}

This is the problem we associate with domains $D_{1}$. In addition to the above description, this domain is assumed to satisfy the following topological conditions:

(i) the domain $D_{1}$ is $\bar{l}_{1}$ - and $\bar{l}_{2^{-}}$convex ${ }^{2}$;

(ii) the projections

$\pi_{1}: \bar{D}_{1} \rightarrow l_{1}$ along the vector field $\bar{l}_{3}$,

$\pi_{2}: \bar{D}_{1} \rightarrow \Gamma$ along the vector field $\bar{l}_{2}$,

$\pi_{3}: \bar{D}_{1} \rightarrow l_{3}$ along the vector field $\bar{l}_{1}$,

satisfy the hypotheses

$$
\pi_{1} D_{1}=O A_{1} \quad \text { and } \quad \pi_{3} D_{1}=O A_{3}
$$

(iii) the curve $\Gamma$ is transversal to the vector field.

The first partly characteristic problem for a given third order strictly hyperbolic differential operator

$$
P(\partial)=\left(\partial / \partial \bar{l}_{1}\right)\left(\partial / \partial \bar{l}_{2}\right)\left(\partial / \bar{l}_{3}\right) u+Q(\partial) u
$$

with $Q(\partial)$ being an arbitrary second order differential operator in $D_{1}$, is formulated in the following way.

Given an arbitrary function $f$ in $\bar{D}_{1}$ and $g$ on $\partial D_{1}=O A_{1} \cup O A_{3} \cup \Gamma$, find a function $u$ in $\bar{D}_{1}$ such that

$$
P(\partial) u=f \quad \text { in } D_{1}, u=g \quad \text { on } \partial D_{1} .
$$

\footnotetext{
${ }^{2}$ For a given smooth vector field $\bar{l}$ in $\mathbb{R}^{n}$ a set $\Omega \subset \mathbb{R}^{n}$ is said to be $\bar{l}$-convex if, for arbitrary points $p$ and $q$ in $\omega$ lying on a trajectory of $\bar{l}$, the full trajectory $(p, q)$ belongs to $\omega$.
} 
Remark The name "characteristic" is used in the title to emphasize that the value of an unknown function $u$ is partially prescribed on the characteristic part $O A_{1} \cup O A_{3}$ of the boundary $\partial D_{1}$.

It is remarkable that even to formulate the result related to problem (58), we need some concepts and notions connected with the dynamical systems considered in Sec. 1.

We introduce two mappings of $\Gamma$ into itself

$$
\delta_{1}=\pi_{2} \circ \pi_{1} \quad \text { and } \quad \delta_{2}=\pi_{2} \circ \pi_{3},
$$

and consider the semigroup $\Phi_{\delta}$ generated by $\delta_{1}$ and $\delta_{2}$. Let $\mathcal{O}_{\delta}$ be the set of orbits in $\Gamma$ corresponding to $\Phi_{\delta}$. Denote by $\mathcal{T}=\mathcal{T}_{1} \cup \mathcal{T}_{2}$ the guiding set on $\Gamma$, consisting of characteristic points in $\Gamma$ with respect to differential operator $P(\partial)$. In other words

$$
q \in \mathcal{T}_{j}, \quad \text { if } \quad T_{q}(\Gamma) \ni \bar{l}_{j}(q), \quad j=1,2,
$$

where $T_{q}(\Gamma)$ stands for the tangent space of $\Gamma$ at the point $q$. Now every thing is ready to introduce the set $\mathfrak{N}_{\delta}^{\mathcal{T}}$ as the collection of all $\mathcal{T}$ - proper periodic orbits lying in the guiding set $\mathcal{T}$.

Example. If the curve $\Gamma$ does not contain any characteristic points (i.e. the tangent space of $\Gamma$ at any point is transversal to all the vectors $\bar{l}_{j}, 1 \leq j \leq 3$ ), then $\mathfrak{N}_{\delta}^{\mathcal{T}}=\emptyset$.

Theorem 21 Assume that the vector fields $\bar{l}_{1}, \bar{l}_{2}$ and $\bar{l}_{3}$ are constant and the characteristic sets $\mathcal{T}_{1}$ and $\mathcal{T}_{2}$ satisfy an ordering condition formulated before Theorem 18. Let $\Gamma$ be a $(2+k)$ - times differentiable curve. Then problem (58) has a unique solution $u \in C^{2+k}\left(\bar{D}_{1}\right)$ for arbitrary functions $f \in C^{k}\left(\bar{D}_{1}\right)$ and $g \in C^{2+k}\left(\partial \bar{D}_{1}\right)$ if and only if $\mathfrak{N}_{\delta}^{\mathcal{T}}=\emptyset$.

Remark. The specifics of this results are of the two different types. First of all, this is the first example of a boundary problem for hyperbolic differential equation in a bounded domain when the value of an unknown function is prescribed on the entire boundary. On the other hand, the boundary problem turns out to be well posed, although it assigns only one boundary condition to a third order differential operator.

Proof. For the sake of brevity we assume the operator $P(\partial)$ to be homogeneous and restrict ourselves to the case $f=0$. It is easily to see that a linear change of variables reduces problem (58) to the problem

$$
\begin{aligned}
\left(r_{1} \partial_{x}+r_{2} \partial_{y}\right) \partial_{x} \partial_{y} u(x, y)=0 & \text { in } D_{1}, \\
u=h & \text { on } \partial D_{1} .
\end{aligned}
$$

The boundary $\partial D$ now consists of three parts,

$$
\begin{gathered}
\Gamma_{1}=\{(x, y) \mid y=0, \quad 0 \leq x \leq 1\}, \quad \Gamma_{2}=\{(x, y) \mid x=0, \quad 0 \leq y \leq 1\}, \\
\Gamma=\left\{(x, y) \mid x=\alpha_{1}(t), \quad y=\alpha_{2}(t) ;-1 \leq t \leq 1\right\},
\end{gathered}
$$

where $\alpha_{1}(-1)=0, \quad \alpha_{1}(1)=1, \quad \alpha_{2}(-1)=-1, \quad \alpha_{2}(1)=0$. Let $h=h_{1}(x)$ on $\Gamma_{1}$, $h=h_{2}(y)$ on $\Gamma_{2}$ and $h=h_{3}(x, y)$ on $\Gamma$. In virtue of continuity of the function $h$ on $\Gamma$, the following compatibility conditions are fulfilled:

$$
h_{1}(0)=h_{2}(0), \quad h_{1}(1)=h_{3}(1,0), \quad h_{2}(1)=h_{3}(0,1) .
$$

Using the postulated properties of the domain $D$ it is easily verified that the function

$$
u(x, y)=\int_{0}^{x}\left(\int_{0}^{y} F\left(r_{2} s-r_{1} t\right) d t\right) d s+h_{1}(x)+h_{2}(y)-h_{1}(0), \quad 0 \leq x, y \leq 1,
$$


satisfies the equation in (59) and the condition $u=h$ on the part $\Gamma_{1} \cup \Gamma_{2}$ of the boundary with any function $F \in C^{1}\left(-r_{1}, r_{2}\right)$. The necessity to satisfy the condition $u=h_{3}$ on $\Gamma$ leads to the integral equation

$$
\int_{0}^{\alpha_{1}(t)}\left(\int_{0}^{\alpha_{2}(t)} F\left(r_{2} x-r_{1} y\right) d y\right) d x=H(t), \quad t \in I .
$$

Here, as in Subsec. 4.2.2, the equalities $x=\alpha_{1}(t), y=\alpha_{2}(t), t \in I$, describe the curve $\Gamma$ in a parametric form, and $H(t)=-h_{1}\left(\alpha_{1}(t)\right)-h_{2}\left(\alpha_{2}(t)\right)+h_{3}\left(\alpha_{1}(t), \alpha_{2}(t)\right)+$ $h_{1}(0)$. What is important is that the function $H$, generated by any $k$ times piecewise differentiable functions $h$, belongs to the space $\mathcal{H}(I)=\left(C^{2} \cap C_{0}\right)(I)$. This follows from (60). The inverse statement is also true: a function $u$ which is defined by formula (61) with $F$ being a solution to equation (62) is a solution of the problem (59). Thus, the latter problem turns out to be equivalent to the integral equation (62), which is nothing but equation (49). The unique solvability of the problem (59) for any function $h$ of the required form under hypothesis $\mathfrak{N}_{\zeta}^{\mathcal{T}}=\emptyset$ follows directly from Theorem 18.

To illustrate the result obtained let us consider the problem (59) in the domains $D_{1}, D_{2}$ and $D_{3}$ (see below). On these figures the only points $p$ and $q$ are charac-

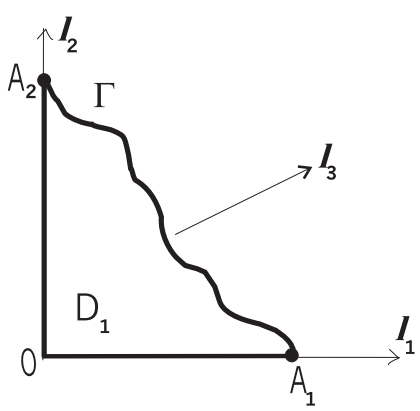

Fig. 3

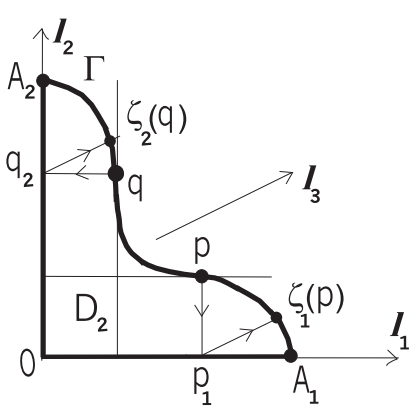

Fig. 4

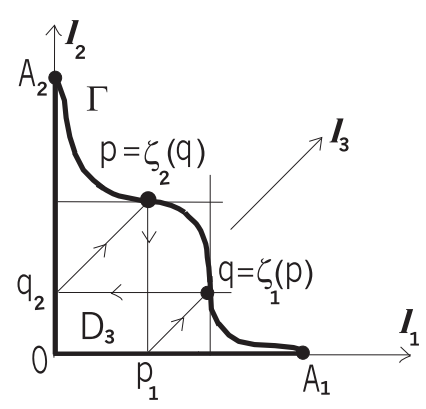

Fig. 5

teristic ones. It is easily verified that $\mathfrak{N}_{\zeta}^{\mathcal{T}}=\emptyset$ in the case of the domains $D_{1}$ and $D_{2}$ and hence, the problem (59) is well posed in $D_{1}$ and in $D_{2}$. On the other hand, the set $\mathfrak{N}_{\zeta}^{\mathcal{T}}$ in the case of $D_{3}$ contains the (unique) characteristic $\mathcal{T}$ - proper periodic orbit $(p, q, p)$. That is why Theorem 2 is nonapplicable here.

\subsubsection{Second partly characteristic problem.}

Let $D_{2}$ be an arbitrary domain associated with $P(\partial)$. Denote by $\Gamma=O O^{\prime}$ a quasi-diagonal of the curvilinear parallelogram $A_{1} O A_{2} O^{\prime}$ which is supposed to be a nonsingular $C^{2}$ - curve satisfying the following topological conditions:

(j) the curve $\Gamma$ is $\bar{l}_{1^{-}}$and $\bar{l}_{2^{-}}$convex;

(jj) the curve $\Gamma$ is transversal to the vector $\bar{l}_{3}$ at any point, and it has no common points with the open intervals $O A_{1}$ and $O A_{2}$.

The second partly characteristic problem for the above differential operator $P(\partial)$ in the domain $D_{2}$ is formulated as follows:

Given arbitrary functions $f$ in $\bar{D}_{2}$ and $g$ on $\mathcal{M}=O A_{1} \cup O A_{2} \cup \Gamma$, find a function $u$, in $\bar{D}_{2}$ such that

$$
\begin{aligned}
P(\partial) u=f & & \text { in } D_{2}, \\
u=g & & \text { on } \mathcal{M} .
\end{aligned}
$$


Remark. By contrast with problem (59), the problem in question is not exactly boundary problem, because a part of the set $\mathcal{M}$ does not lie in $\partial D_{2}$.

Proceeding with the formulation of the result related to problem (63) we first establish some compatibility conditions which must hold for the function $g$. Denote by $g_{1}, g_{2}$ and $g_{3}$ the restrictions of the function $g$ to the curves $O A_{1}, O A_{2}$ and $\Gamma$, respectively. If $\left\{x=x_{j}(t), y=y_{j}(t) ; 0 \leq t \leq 1\right\}, j=1,2,3$, are some smooth parametric representation of these curves and $O=\left(x_{j}(0), y_{j}(0)\right)$ for all $j$, then

$$
u\left(x_{j}(t), y_{j}(t)\right)=g_{j}(t), 0 \leq t \leq 1
$$

for $j=1,2,3$. Denote by $\bar{\tau}_{\Gamma}$ the unit tangent vector of $\Gamma$ at the point $O$. By virtue of transversality of the vectors $\bar{l}_{1}$ and $\bar{l}_{2}$, there are positive constants $\lambda_{1}$ and $\lambda_{2}$ such that

$$
\bar{\tau}_{\Gamma}=\lambda_{1} \bar{l}_{1}+\lambda_{2} \bar{l}_{2} .
$$

This immediately leads to the first compatibility condition

$$
g_{3}^{\prime}(O)=\lambda_{1} g_{1}^{\prime}(O)+\lambda_{2} g_{2}^{\prime}(O)
$$

The second one

$$
g_{1}(O)=g_{2}(O)=g_{3}(O)=u(O)
$$

is a consequence of the coincidence of the curves $O A_{1}, O A_{2}$ and $\Gamma$ at the point $O$.

Theorem 22 Let $P(\partial)$ be the above differential operator with constant vector fields $\bar{l}_{j}, 1 \leq j \leq 3$, defined in a domain $D_{2}$. Then, for arbitrary functions $f \in C\left(\bar{D}_{2}\right)$ and $g \in C^{2}(\mathcal{M})$ satisfying conditions (64) and (65), there is a unique solution $u \in C^{2}\left(\bar{D}_{1}\right)$ of problem (63), and the inverse operator $(f, g) \mapsto u$ is bounded. If $f \in C^{k}\left(\bar{D}_{1}\right)$ and $g \in C^{2+k}(\mathcal{M})$, then $u \in C^{2+k}\left(\bar{D}_{1}\right), k=1,2, \ldots$

The relation $g \in C^{m}(\mathcal{M})$ means that the three functions $g_{1}, g_{2}$ and $g_{3}$ are $m$ times continuously differentiable on their domains.

Proof. For the sake of brevity, we consider only the case of a homogeneous operator $P(\partial)$ (i.e. $Q \equiv 0)$. In this situation there is a linear change of variable reducing problem (63) to the problem

$$
\begin{aligned}
\left(\partial_{x}-\partial_{y}\right) \partial_{x} \partial_{y} u=f & & \text { in } D_{2} \\
u=g & & \text { on } \mathcal{M}
\end{aligned}
$$

where

$$
D_{2}=\{(x, y) \mid 0 \leq x \leq X, 0 \leq y \leq Y\}
$$

and the set $\mathcal{M}$ consist of the three parts,

$$
\mathcal{M}_{1}=\{(x, y) \mid 0 \leq x \leq X, y=0\}, \quad \mathcal{M}_{2}=\{(x, y) \mid x=0,0 \leq y \leq Y\},
$$

and

$$
\Gamma=\left\{(x, y) \mid x=\delta_{1}(t), y=\delta_{2}(t), 0 \leq t \leq 1\right\} .
$$

The relations $x=\delta_{1}(t), y=\delta_{2}(t)$ determine a parametric representation of the curve $\Gamma$, and the functions $\delta_{1}$ and $\delta_{2}$ satisfy the following conditions dictated by geometric properties of $\Gamma$ :

$$
\begin{aligned}
& \delta_{1}(0)=\delta_{2}(0)=0 ; \quad\left(\delta_{1}(1), \delta_{2}(1)\right)=(X, Y) ; \\
& \delta_{1}(t) \delta_{2}(t)>0, \quad 0<t \leq 1 ; \quad \delta_{1}^{\prime}(0) \delta_{2}^{\prime}(0)>0 \\
& \delta_{1}^{\prime}(t)+\delta_{2}^{\prime}(t)>0 \quad \text { and } \quad \delta_{1}^{\prime}(t) \delta_{2}^{\prime}(t) \geq 0 .
\end{aligned}
$$


To see this it is necessary to take into account that the properties (i) and (ii) of $\Gamma$ postulated above remain valid under linear nonsingular transformations in $\mathbb{R}^{2}$. The relations (k) are obvious. The (kk) follows from the properties (i) and (ii) of $\Gamma$, and finally, the relations (kkk) are nothing other than the property (i) of $\Gamma$. Indeed, as we have now $\bar{l}_{1}=(1,0), \bar{l}_{2}=(0,1)$ and $\bar{l}_{3}=(1,-1)$, the first inequality in $(\mathrm{kkk})$ is equivalent to the transversality of an arbitrary tangent vector $\bar{\tau}(t)=\left(\delta_{1}^{\prime}(t), \delta_{2}^{\prime}(t)\right)$ of $\Gamma$ to the vector $\bar{l}_{3}$, whereas the second one follows from (i). The first relation in (kkk) makes possible to assume (without loss of generality) that the functions $\delta_{1}(t)$ and $\delta_{2}(t)$ satisfy the condition

$$
(k)^{\prime} \quad \delta_{1}(t)+\delta_{2}(t)=t
$$

Let

$$
g=G_{1}(x) \text { on } \mathcal{M}_{1}, \quad g=G_{2}(y) \text { on } \mathcal{M}_{3}, \quad g=G_{3}(x, y) \text { on } \Gamma .
$$

Introduce the function $\varphi(t)=G_{3}\left(\delta_{1}(t), \delta_{2}(t)\right), 0 \leq t \leq 1$. The first necessary compatibility condition related to the functions $G_{1}, G_{2}$ and $\varphi$ is, by (65),

$$
G_{1}(0)=G_{2}(0)=\varphi(0) .
$$

To formulate condition (64) corresponding to the operator $\left(\partial_{x}-\partial_{y}\right) \partial_{x} \partial_{y}$ we note that now $\bar{l}_{1}=(1,0), \bar{l}_{2}=(0,1)$ and $\bar{\tau}_{\Gamma}=\left(\delta_{1}^{\prime}(0), \delta_{2}^{\prime}(0)\right)$. This means that the coefficients $\lambda_{1}$ and $\lambda_{2}$ in (64) are now $\delta_{1}^{\prime}(0)$ and $\delta_{2}^{\prime}(0)$, respectively. Hence, the second compatibility condition has a form

$$
\varphi^{\prime}(0)=\delta_{1}^{\prime}(0) G_{1}^{\prime}(0)+\delta_{2}^{\prime}(0) G_{2}^{\prime}(0) .
$$

As the first step in the proof of Theorem 2 we will establish its validity in the case $f=0$. Introduce the function

$$
u(x, y)=\int_{0}^{x}\left(\int_{0}^{y} F(s+t) d t\right) d s+G_{1}(x)+G_{2}(y)-G_{1}(0)
$$

in $D_{2}$, where $F$ is an arbitrary continuous function in the closed interval $I_{r}=$ $(0, X+Y)$. By virtue of $(67)$, the function $u(x, y)$ satisfies the boundary conditions $u=g$ on $\mathcal{M}_{1}$ and $\mathcal{M}_{2}$. On the other hand, this function solves the homogeneous equation in (66) (if the operator $\partial_{x}-\partial_{y}$ to treat as a vector field $(1,-1) \cdot\left(\partial_{x}, \partial_{y}\right)$ ).

To solve problem (66) with $f=0$ it remains to choose a continuous function $F$ such that the function $u(x, y)$ in question satisfies the condition

$$
u(x, y)=G_{3}(x, y) \quad \text { on } \Gamma .
$$

Thus, we arrive at the integral equation

$$
\int_{0}^{\delta_{1}(t)}\left(\int_{0}^{\delta_{2}(t)} F(x+y) d y\right) d x=H(t), \quad 0 \leq t \leq 1
$$

for an unknown function $F \in C\left(I_{r}\right)$. Here

$$
H(t)=-G_{1}\left(\delta_{1}(t)\right)-G_{2}\left(\delta_{2}(t)\right)+G_{3}\left(\delta_{1}(t), \delta_{2}(t)\right)+G_{1}(0) .
$$

What is important is that the function $H(t)$ satisfies the conditions

$$
H(0)=0 \quad \text { and } \quad H^{\prime}(0)=0 .
$$

This follows immediately from compatibility conditions (67) and (68). But then equation (69) coincides with equation (49) whose solvability has been demonstrated 
in the very end of the proof of Theorem 17 . This completes the proof of Theorem 22 when $f=0$. In the case of an arbitrary function $f \in C^{k}\left(\bar{D}_{2}\right)$ what it remains to do is to find a particular solution $u \in C^{2+k}\left(\bar{D}_{1}\right)$ of the differential equation

$$
\left(\partial_{x}-\partial_{y}\right) \partial_{x} \partial_{y} u=f, \quad(x, y) \in D_{2} .
$$

The reader can verify directly that the function

$$
u(x, y)=\int_{0}^{x}\left(\int_{0}^{y}\left(\int_{\gamma(s+t)}^{s-t} f\left(\frac{s+t+z}{2}, \frac{s+t-z}{2}\right) d z\right) d t\right) d s
$$

with $\gamma=\left(\delta_{1}-\delta_{2}\right) \circ\left(\delta_{1}+\delta_{2}\right)^{-1}$, is a particular solution of this equation in the domain $D_{2}$. The assertion of the theorem is proved.

\section{References}

[Ch] C. Chester, Techniques in PDE, Mc.Graw - Hill Book Company, (1972)

[KCG] M.Kuczma, B.Choczewski and R.Ger, Iterative functional equations, Encyclopedia of Mathematics and its applications 32, Cambridge University Press, Cambridge, 1990.

[GGV] I.Gelfand, M.Graev and N.Vilenkin, Integral Geometry and Representation Theory, New York, Academic Press, 1966.

[P1] B.Paneah, On Solvability of Functional Equations Relating to Dynamical System with Two Generators, Functional Analysis and Its Applications, v.37, No.1, pp.46-60, 2003.

[P2] B.Paneah, Dynamical Approach to Some Problems in Integral Geometry, Trans.Amer.Math.Soc., v.356, No.7, pp.2757-2780, 2003.

[P3] B.Paneah, On a Problem in Integral Geometry connected to the Dirichlet Problem for Hyperbolic Equations, International Mathematical Research Notes (IMRN), (1997), No.5, $213-222$.

[P4] B.Paneah, Dynamic Methods in the General Theory of Cauchy Type Functional Equations, Contemporary Mathematics,v.364, pp.205 - 223, 2004.

[P5] B.Paneah, Noncommutative Dynamical Systems with Two Generators and Their Applications in Analysis, Discret and Continuous Dynamical Systems, v.9, No.6, pp.1411-1420, 2003.

[Z] M.Zdun, On the Uniqueness of Solutions of the Functional Equation $\varphi(x+f(x))=\varphi(x)+\varphi(f(x))$, Aequationes Math. (1972), 8, 229 - 232.

DEPARTMENT OF MATHEMATICS, TECHNION

HAIFA, ISRAEL

e-mail: peter@techunix.technion.ac.il 